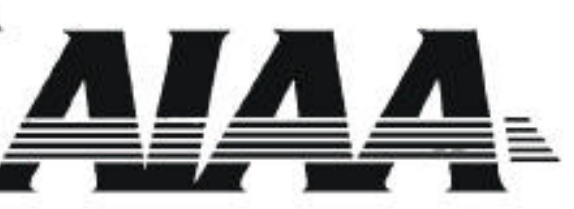

AIAA-2000-2315

DRAG REDUCTION OF AIRFOILS WITH MINIFLAPS. CAN WE LEARN FROM DRAGONFLIES?

D.W. Bechert, R. Meyer and W. Hage

DLR, Department of Turbulence Research,

Mueller-Breslau-Str. 8, D-10623 Berlin, Germany

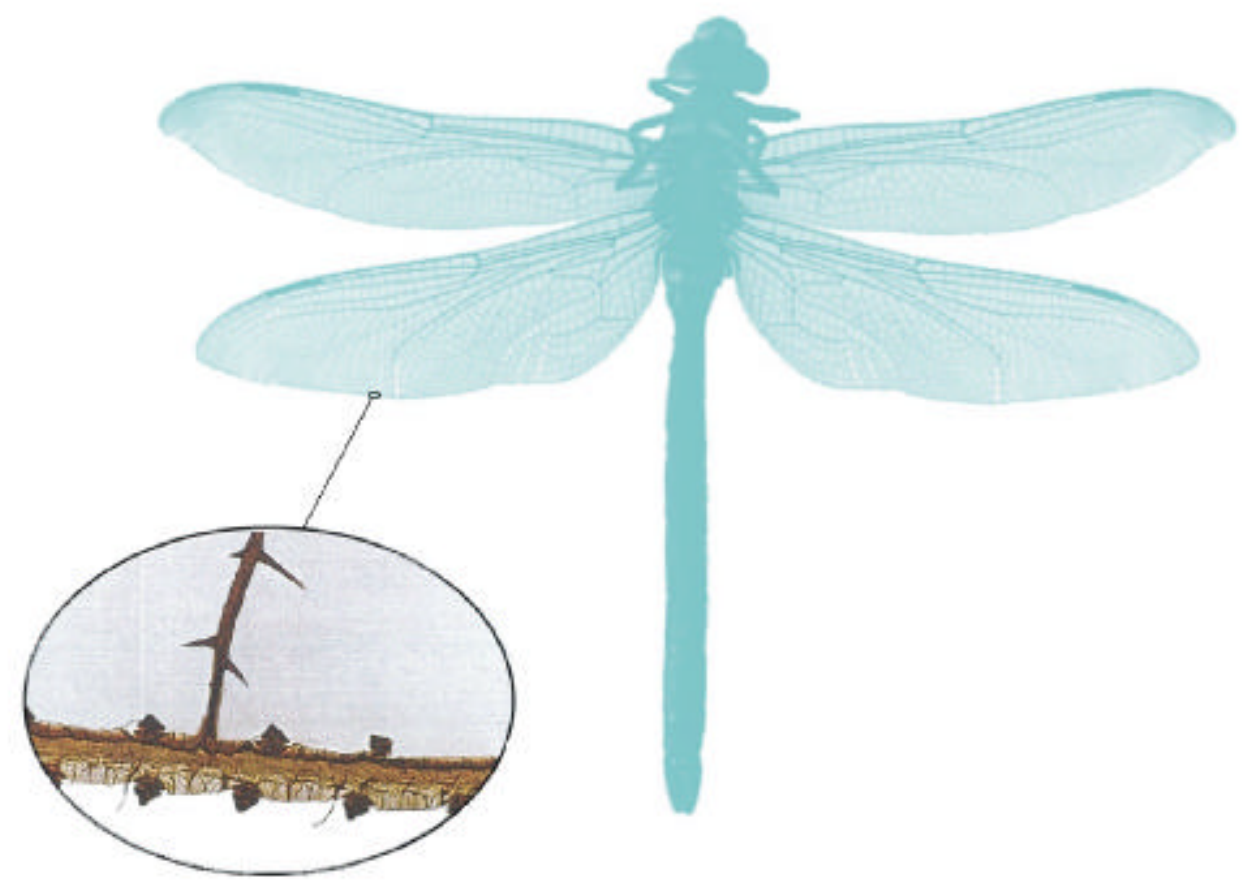

Fluids 2000

19-22 June 2000 / Denver, CO

For permission to copy or republish, contact the American Institute of Aeronautics and Astronautics 1801 Alexander Bell Drive, Suite 500, Reston, VA 20191 


\title{
DRAG REDUCTION OF AIRFOILS WITH MINIFLAPS. CAN WE LEARN FROM DRAGONFLIES?
}

\author{
D. W. Bechert, R. Meyer and W. Hage \\ DLR, Department of Turbulence Research, \\ Mueller-Breslau-Str. 8, 10623 Berlin, Germany
}

\begin{abstract}
.
Miniflaps at the trailing edges of airfoils (e.g., Gurney flaps or divergent trailing edges) change the Kutta condition and thus produce higher lift. Unfortunately, however, the drag is also increased due to the flow separation downstream of this particular type of trailing edge. Therefore, the trade-off between beneficial and detrimental effects is considered in this paper. Various aspects of the flow on airfoils with Gurney flaps are addressed:
\end{abstract}

(1) Transonic flow. Wind tunnel experiments have been carried out with a CAST 10-2/DOA2 airfoil with Gurney flap and at high subsonic flow speed. The lift to drag ratio is improved and the test wing behaves like one having a $20 \%$ larger surface area. In addition, buffeting becomes less critical.

(2) Selection of flap size. Detailed wind tunnel studies have been carried out with a low-drag glider wing at low subsonic velocities. The Gurney flap height was varied in six steps so that the most relevant parameter regime was covered. For the lift increase and for the device drag, simple empirical laws were obtained. Subsequently, for practical applications, a procedure for the selection of a suitable flap height providing a beneficial effect has been devised.

(3) Drag reduction by wake stabilization. In the separation regime downstream of a Gurney flap, an absolute instability, i.e., a Karman vortex street occurs, even if the incident boundary layers are turbulent. Therefore, one approach towards drag reduction is to stabilize the wake and hence eliminate the Karman vortex street. This can be achieved with a variety of trailing edge modifications, e.g., with slits or holes in the Gurney flap. A particular structure which exhibits spadelike protrusions at the trailing edge produces also good results. Actually, the latter structure has been adopted from the trailing edge of dragonfly wings. Eliminating the Karman vortex street with these various trailing edge modifications reduces the Gurney flap device drag by about $22-30 \%$ without a perceivable change of the enhanced lift. Obviously, vibration and noise radiation are also reduced together with the suppression of the Karman vortex street.

(4) Drag reduction with a "wakebody". The separation regime downstream of the Gurney flap is not removed by merely stabilizing the wake flow. However, filling this regime with a tapered (twodimensional) body does produce attached flow. With this device, termed a "wake-body" it is possible to further reduce the Gurney flap device drag by more than $48 \%$.

Copyright (C) 2000 by D. W. Bechert, R. Meyer \& W. Hage. Published by the American Institute of Aeronautics and Astronautics, Inc. With permission. 


\section{CONTENTS}

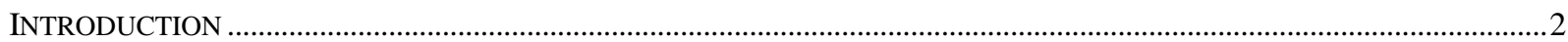

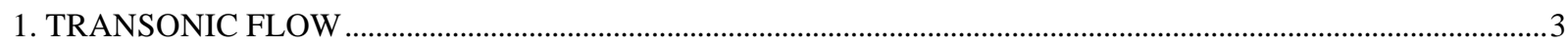

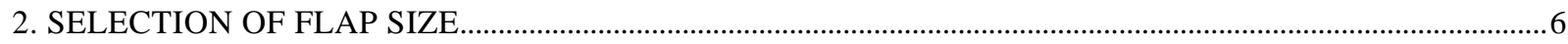

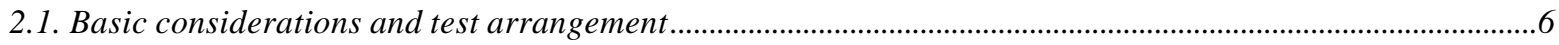

2.2. Experiments with two-dimensional trailing edge geometries ..........................................................................8

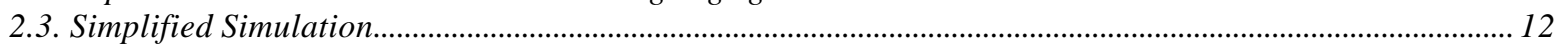

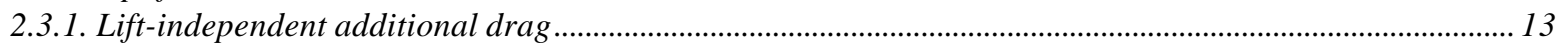

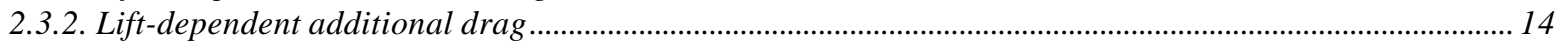

2.3.3. Combined additional drag: lift-independent and lift-dependent .............................................................. 15

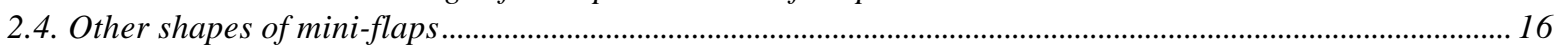

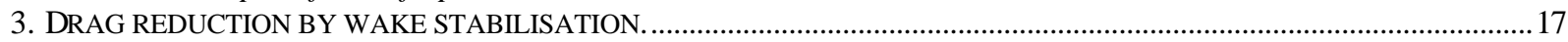

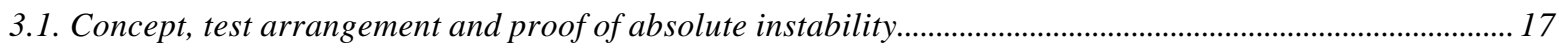

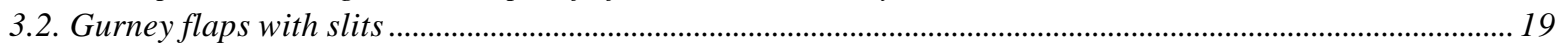

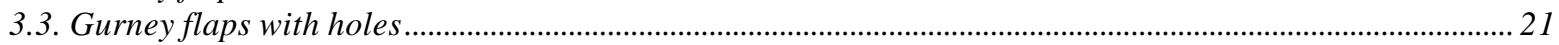

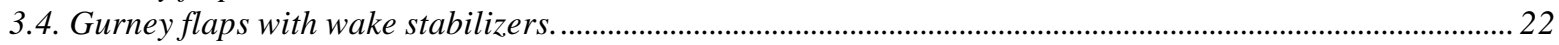

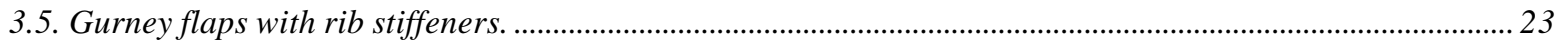

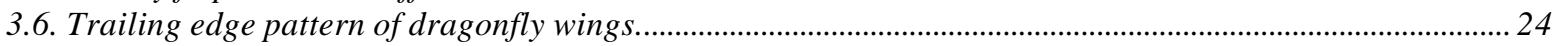

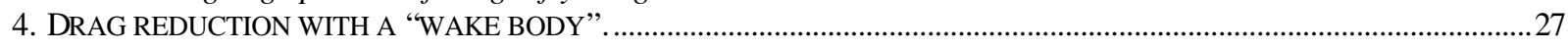

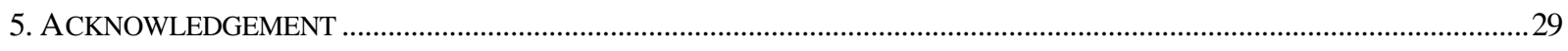

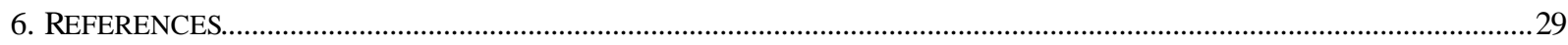

\section{Introduction}

Miniflaps such as Gurney flaps are small extensions at the trailing edge of an airfoil which point downwards at an angle of about $90^{\circ}$. The size is typically between $0.5 \%$ and $2 \%$ of the airfoil chord length. These very small flaps enhance the lift of an airfoil considerably, typically by 6-25\%. These devices have been first used in the seventies on racing cars by Dan Gurney. Since that time, they are attached to the rear wings on those vehicles, producing enhanced negative lift. That causes better road contact and hence permits increased speed on road bends. After Gurney's invention for racing cars, Liebeck [1] recognized the potential of Gurney flaps for aircraft wings. This led to further investigations at McDonnell Douglas on modified trailing edges, also referred to as divergent trailing edges (DTE). Henne [2] has given a detailed survey on this work and on previous related ideas. The commercial transport airliner MD11 is the first aircraft equipped with a diverging trailing edge which permits an improved performance at transonic cruise conditions.

The shape of the trailing edge is of paramount importance for the lift. In addition, it is worth mentioning (see also Henne [2]) that the static pressure on the lower side upstream of the Gurney flap or the diverging trailing edge is significantly higher than the value which is found at the end of the upper side of the airfoil. Consequently, traditional closure principles such as the condition of the continuity of static pressure at the trailing edge have to be abandoned here. Recent detailed investigations on the flow on diverging trailing edges have shed some light on the local flow structure [3-5]. At present, further technological applications of miniflaps on aircraft for high lift conditions [6] and for transonic cruise [2] are likely. Enhanced performance of wind turbines has also been demonstrated [7]. Numerous other applications are conceivable such as, e.g., in turbomachines. 


\section{TRANSONIC FLOW}

Investigations on the effect of Gurney flaps on the transonic airfoil CAST 10-2/DOA2 have been carried out in the DLR transonic wind tunnel in Göttingen. This airfoil was designed for a Mach number of 0.765 and an angle of attack of $2^{\circ}$. The geometrical configuration of the airfoil and of the attached Gurney flaps can be seen in Fig. 1. The wind tunnel data shown here have been collected by pressure distribution (lift) and by wake rake (drag) measurements. The experiment was essentially twodimensional with the (unswept) wing spanning the whole width of the quadratic $1 \times 1 \mathrm{~m}^{2}$ test section of the wind tunnel. The wind tunnel wall was perforated in order to minimize wall effects and to permit comparisons with a previous comprehensive data set [8] which had been collected with the same wing in the same wind tunnel configuration.

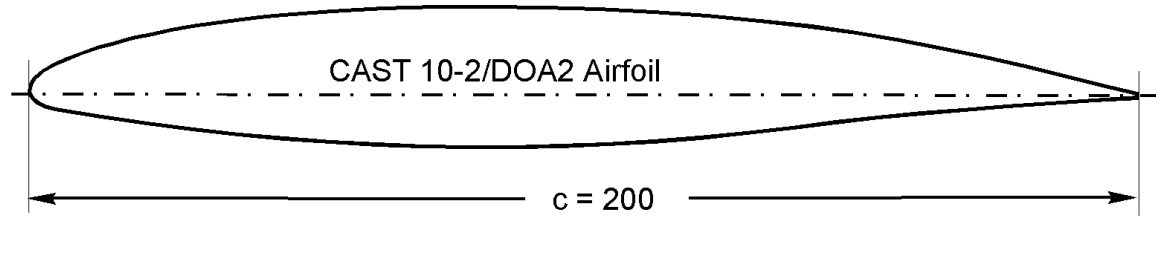

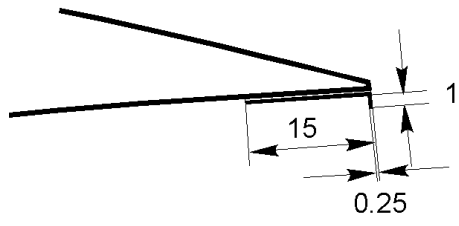

(a) Gurney flap $0.5 \% \mathrm{c}$

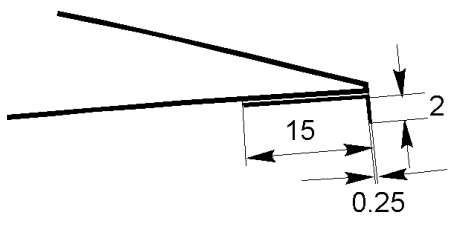

(b) Gurney flap $1 \% \mathrm{c}$

Fig. 1. Test wing with CAST 10-2/DOA2 airfoil with either (a) a flap height of $0.5 \%$ chord or (b) $1 \%$ chord. Dimensions in mm. Adhesive film and Gurney flaps each $0.25 \mathrm{~mm}$ thick.

In Fig. 2, it can be seen that the tiny Gurney flaps cause large effects on both lift and lift to drag ratio. It is interesting to see that even with a Gurney flap height of $0.5 \%$ of the chord length the lift increase persists whereas the parasitic drag caused by the separation downstream of the flap is reduced according to the reduction of the device height form $1 \%$ to $0.5 \%$ chord. Thus, one obvious way to reduce the parasitic drag of Gurney flaps consists of the selection of an optimal, and, actually very small, height of the device.
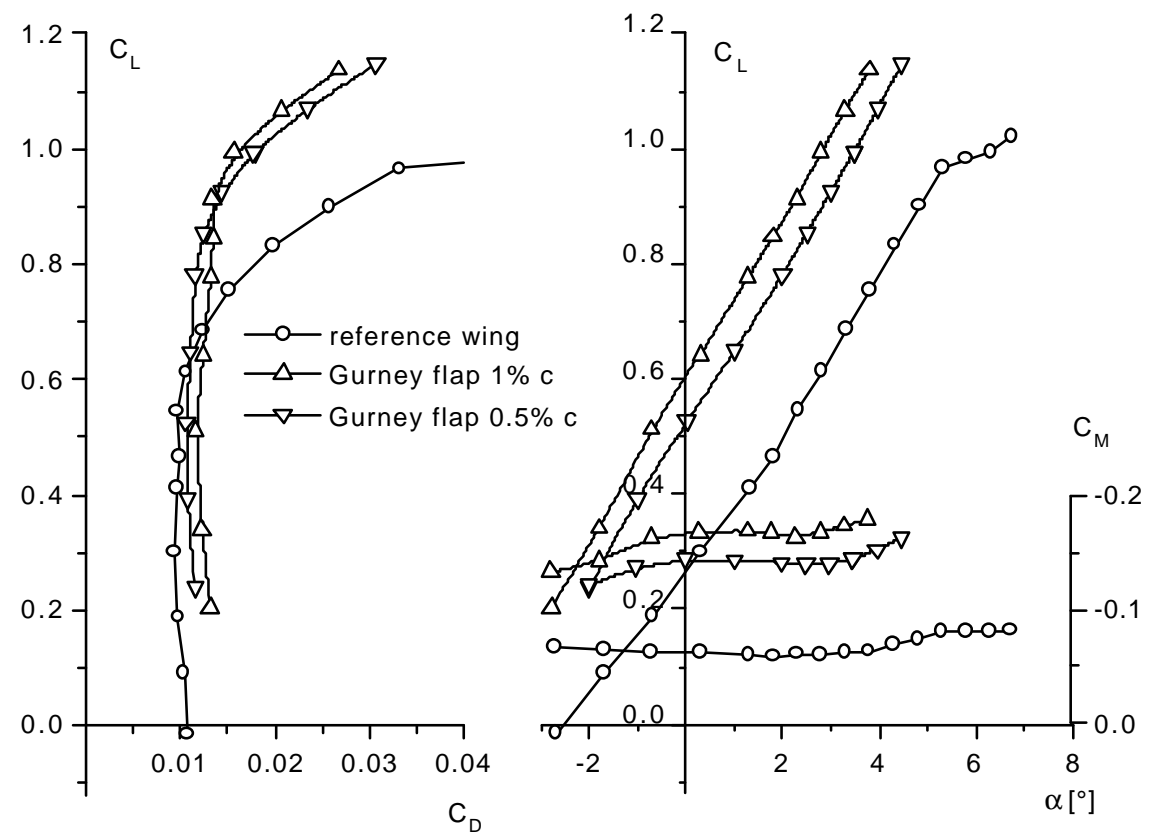

Fig. 2. Drag polars, lift coefficient $C_{L}$ and moment coefficient $C_{M}$ plotted versus the angle of attack $\alpha$. Airfoil CAST 10-2/DOA2, Mach number $M=0.73$, Reynolds number $\operatorname{Re}=2.7 \times 10^{6}$. Data from [9]. 
In Fig. 3, we show pressure distributions without and with Gurney flap. The lift coefficient is at about $\mathrm{C}_{\mathrm{L}} \approx 0.9$ where differences between the two cases are most obvious. Due to the presence of the Gurney flap, the angle of attack at which this lift coefficient is obtained, is lower. As a consequence thereof, the local supersonic Mach numbers on the forward part of the upper side of the airfoil are lower. This ensues a weaker shock at the downstream end of that supersonic flow regime. Thus, the drag is significantly reduced.

One may summarize the properties of the altered wing as being comparable to one having an about $20 \%$ increased wing area. In addition, this altered wing has an improved lift to drag ratio. The beneficial effects in this particular case are mostly due to compressibility. With increasing supersonic Mach numbers on the upper side of the airfoil, it becomes increasingly difficult to keep shock strengths and ensuing separation losses at a low level. Thus, enhancing the loading of the lower surface with a Gurney flap (or a diverging trailing edge) helps to reduce those losses. However, this requires a subtle design of the airfoil and its trailing edge.

In our experiments with various modifications of the test wing, vibrations (buffeting) of the wing always started to occur at about the same angle of attack for a given Mach number. However, for a given angle of attack, the lift with Gurney flap is significantly higher that without. This means that with a Gurney flap (or DTE) in place, the buffeting limit is shifted away from the cruise conditions of a commercial aircraft flying at high subsonic speeds. 

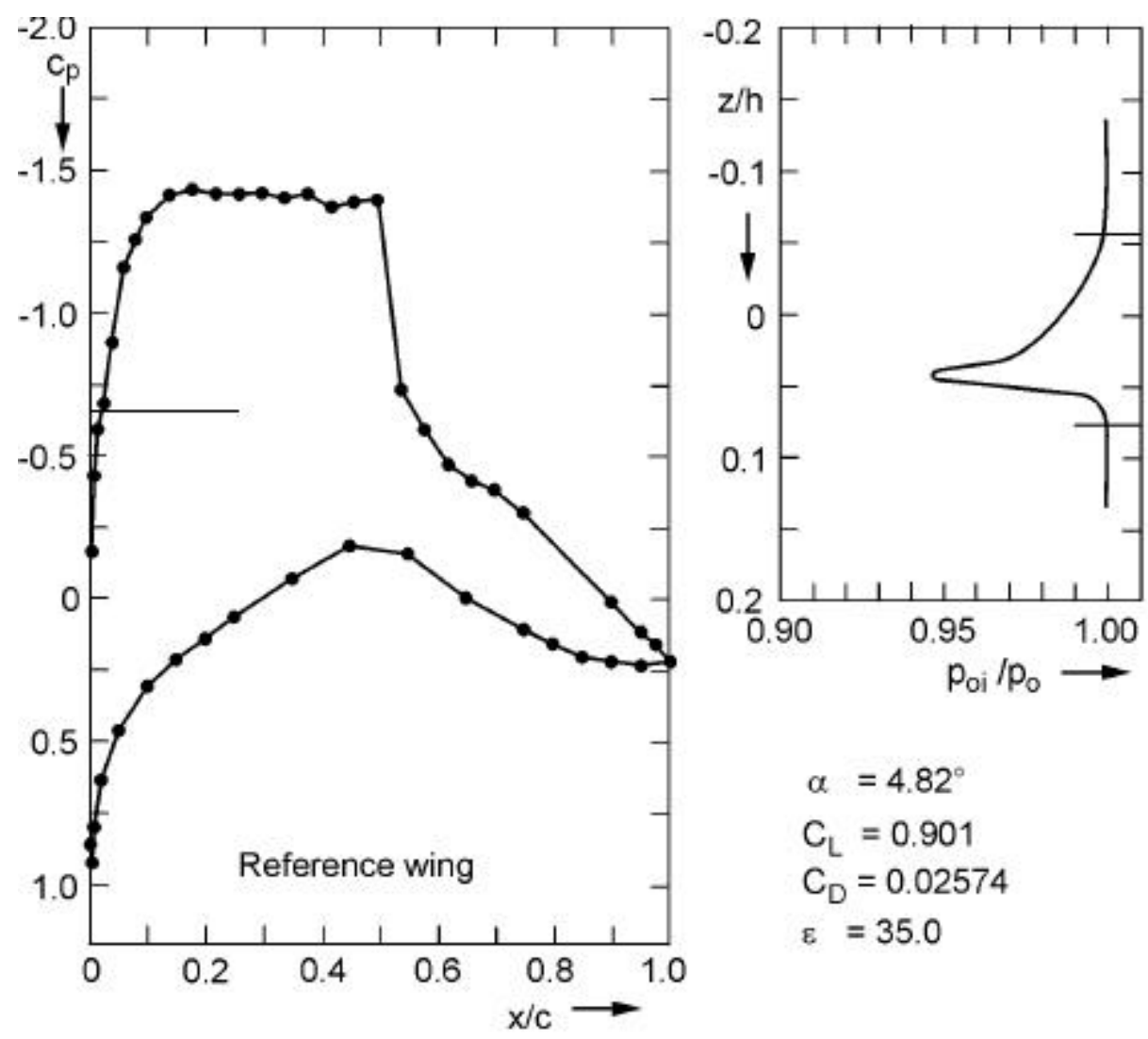

$$
\begin{aligned}
& \alpha=4.82^{\circ} \\
& C_{L}=0.901 \\
& C_{D}=0.02574 \\
& \varepsilon=35.0
\end{aligned}
$$
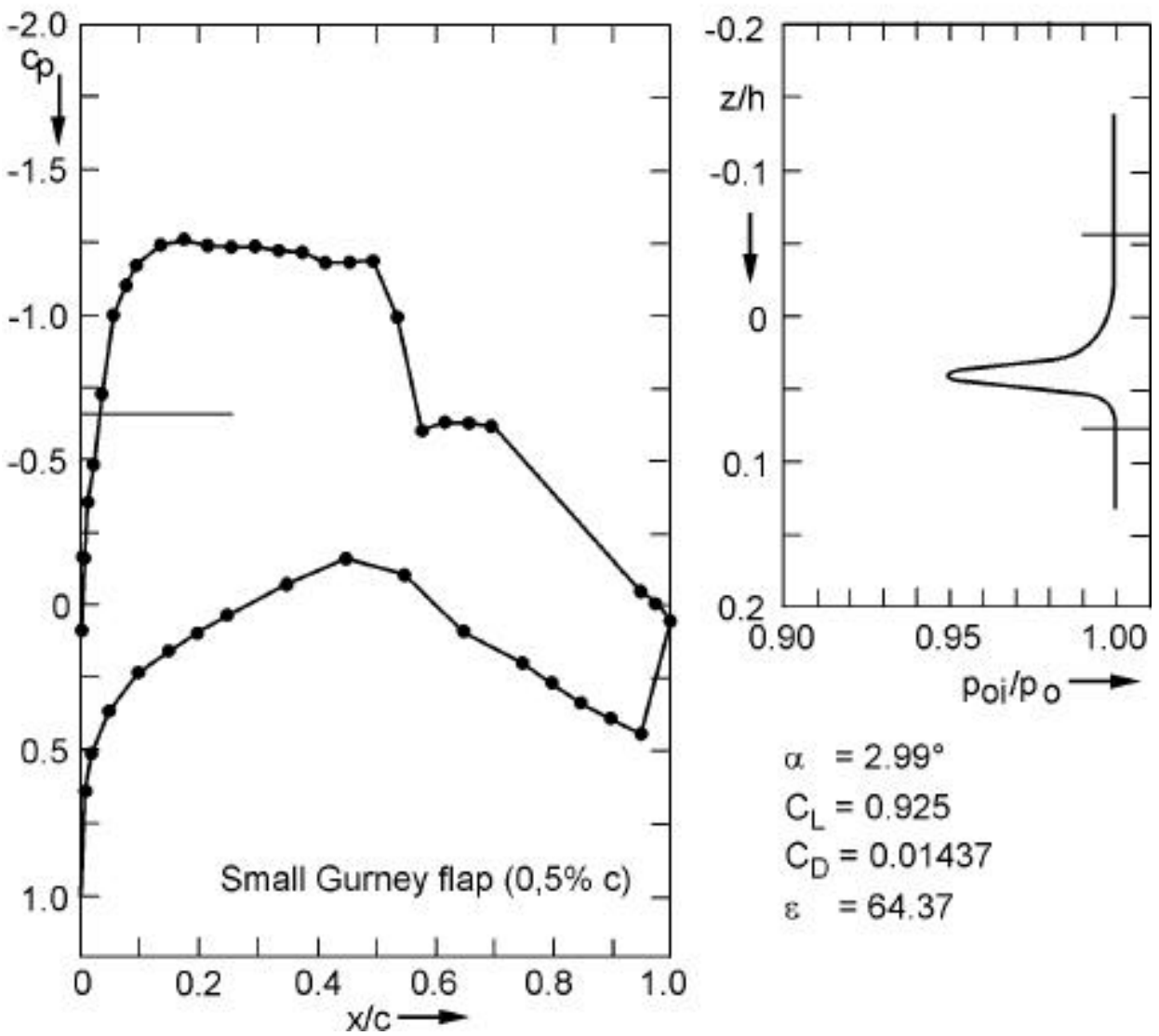

$$
\begin{aligned}
& \alpha=2.99^{\circ} \\
& C_{L}=0.925 \\
& C_{D}=0.01437 \\
& \varepsilon=64.37
\end{aligned}
$$

Fig. 3. Pressure distribution and wake profile of reference wing and wing with small Gurney flap $(0.5 \%)$. Airfoil, Mach and Reynolds numbers as in Fig. 2. Data from [9]. $\varepsilon$ is the lift to drag ratio $\varepsilon=C_{L} / C_{D}$ 


\section{SELECTION OF FLAP SIZE}

\subsection{Basic considerations and test arrangement}

The aim of the experiments described below is to find ways to minimize the parasitic drag of Gurney flaps. This does not necessarily require experiments at transonic speeds. Therefore, these investigations have been carried out in a slow-speed wind tunnel $(\mathrm{M} \approx 0.1)$ with low operating costs and easy access, i.e., the wind tunnel at the Technical University of Berlin. For the tests, a wing with a laminar airfoil was selected, because the low drag of these airfoils enables us to detect conveniently even incremental changes of the parasitic drag of various types of Gurney flaps. The HQ17 airfoil being used had been designed by Horstmann and Quast from DLR for application on glider aircraft. The airfoil co-ordinates are given in a previous paper [10]. The lift and drag data in this second test series were collected by direct force measurements with a 6-component strain gauge wind tunnel balance with high resolution. Some of the Gurney flap devices being tested have a three-dimensional shape. Thus, it was assumed that here, direct force measurements should produce more reliable data than a wake rake would.

The arrangement of the test wing in the wind tunnel test section with rectangular cross section $(1400 \mathrm{x}$ $2000 \mathrm{~mm}^{2}$ ) can be seen in Fig. 4. The test wing is mounted between side plates which extend between floor and ceiling of the test section. The advantage of the side plates is that the boundary layer on their surface is thinner than the one which develops on the wind tunnel walls. On the other hand, one has to deal with variable bypass flow rates between side plates and wind tunnel walls due to variable blockage effects caused by the test wing operating at variable angles of attack. By measuring the bypass flow rates we took into account this influence on the velocity incident on the wing. In addition to that, the usual wind tunnel corrections [11] have been applied.

Nevertheless, there are additional side plate effects which contaminate in particular the drag force measurements. Even with the side plates extending over the whole height of the test section, we cannot exclude that there is still a residual contribution of induced drag. In addition, the laminar flow regimes on the wing are locally contaminated by turbulent "wedges" caused by the turbulent flow on the side plates. Further, there is a small leakage flow in the gap between wing and side plates. However, due to the narrow width of that gap $(0.2 \mathrm{~mm})$ that influence is considered as not very important.

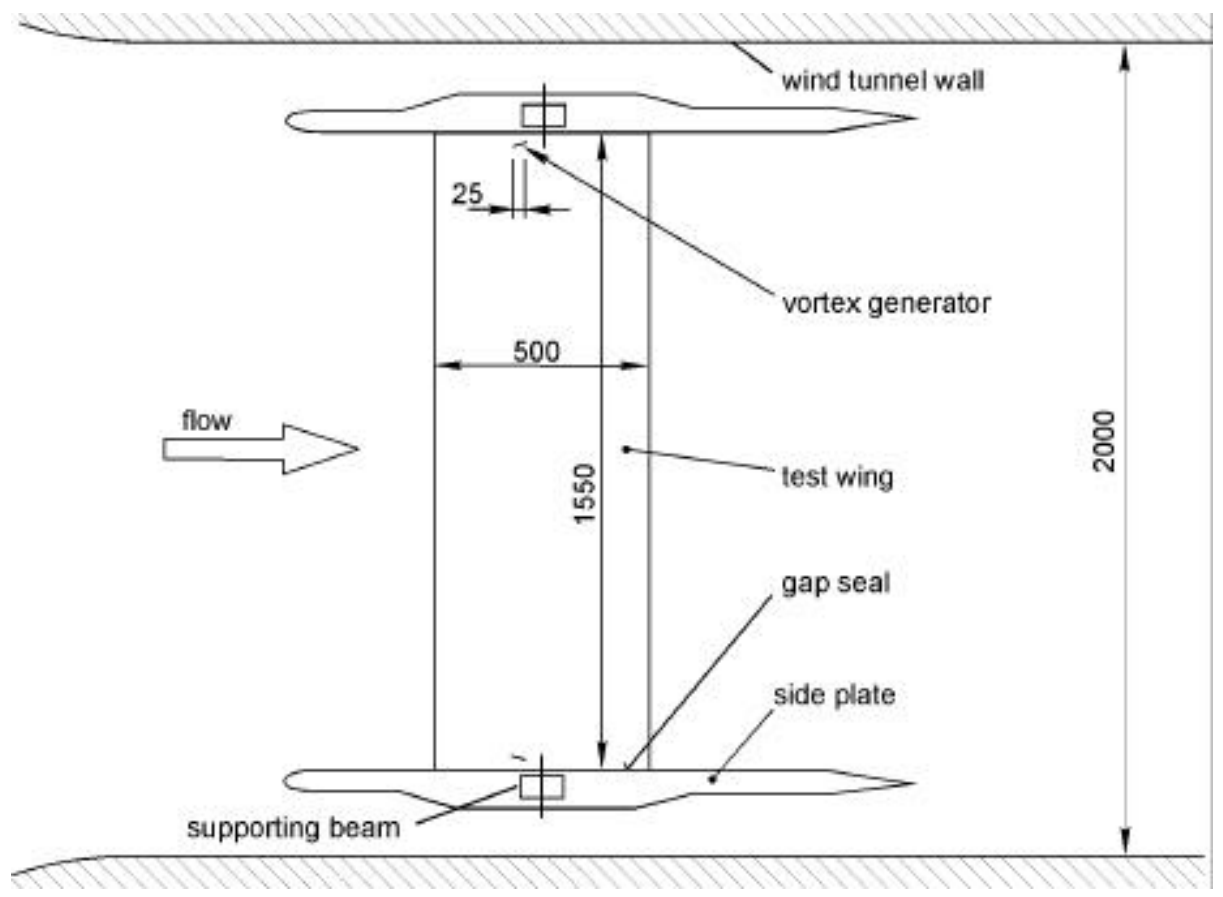

Fig. 4. Wing installation in the test section of the wind tunnel. Dimensions in $\mathrm{mm}$. 
On the other hand, flow vizualization shows that local flow separation occurs in the corners between wing and side plates. These separation regimes could be eliminated by vortex generators on the wing, see Fig. 4. That reduced the total drag of the wing typically by about $10 \%$. Obviously, under these circumstances, our wing cannot exhibit the low drag values which had been obtained by previous wake rake measurements in Delft [12] and Stuttgart [13]. A comparison of these data is shown in Fig. 5.

In our measurements, we used a turbulator on the lower side of the airfoil at $65 \%$ chord. It consisted of a plastic zigzag strip with $0.75 \mathrm{~mm}$ thickness. Location and thickness were determined experimentally based on flow visualisation with paint on the lower airfoil surface. This particular parameter choice eliminated the laminar separation bubble there for the Reynolds numbers at which we collected data, i.e., for 0.5 and $1 \times 10^{6}$. In addition, a small drag reduction was observed. The use of a turbulator seemed important because the boundary layer incident on the Gurney flap should be well-defined. We also checked that the transition on the lower side of the airfoil did not perceivably move once a Gurney flap was installed.

The aim of the present investigation, however, is not to "streamline" data so that all differences vanish. We want to investigate trailing edge modifications on a wing with low drag. For this purpose, the curve (a) in Fig. 5 suits us well as a reference drag polar.
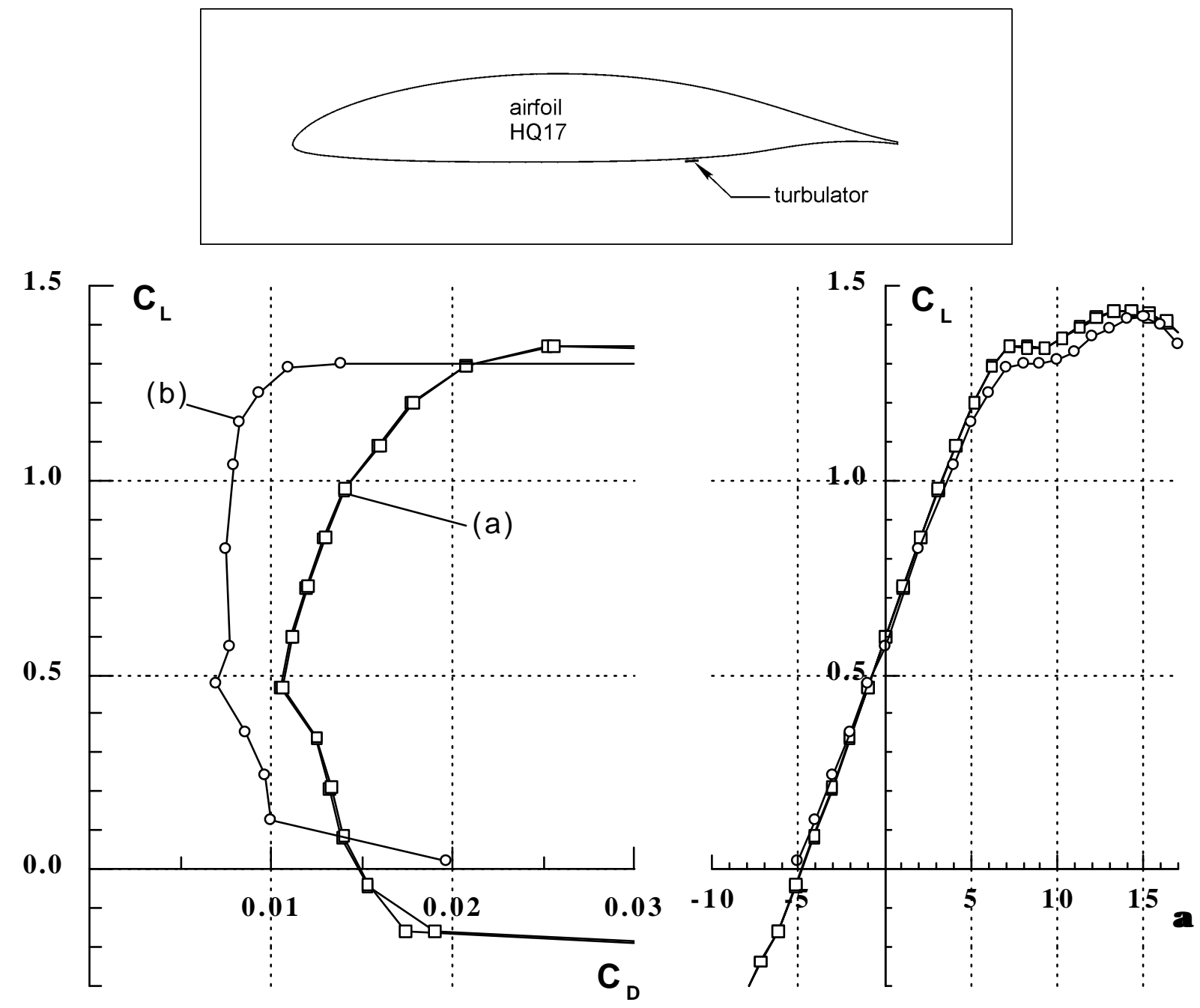

Fig. 5. Comparison of present direct force measurements (a) with previous data [15-17], based on pressure distribution and wake-rake measurements (b). Airfoil: HQ17, $\operatorname{Re}=1 \times 10^{6}$. 


\subsection{Experiments with two-dimensional trailing edge geometries}

In order to obtain more information on a suitable size of a Gurney flap, we have carried out wind tunnel tests with different heights of those devices. Data for $\operatorname{Re}=0.5 \times 10^{6}$ and $\operatorname{Re}=1.0 \times 10^{6}$ can be seen in Figures 6 and 7. We have chosen the particular trailing edge configuration of Figures 6 and 7 in order to produce a somewhat tapered shape in front of the vertical part of the flap.
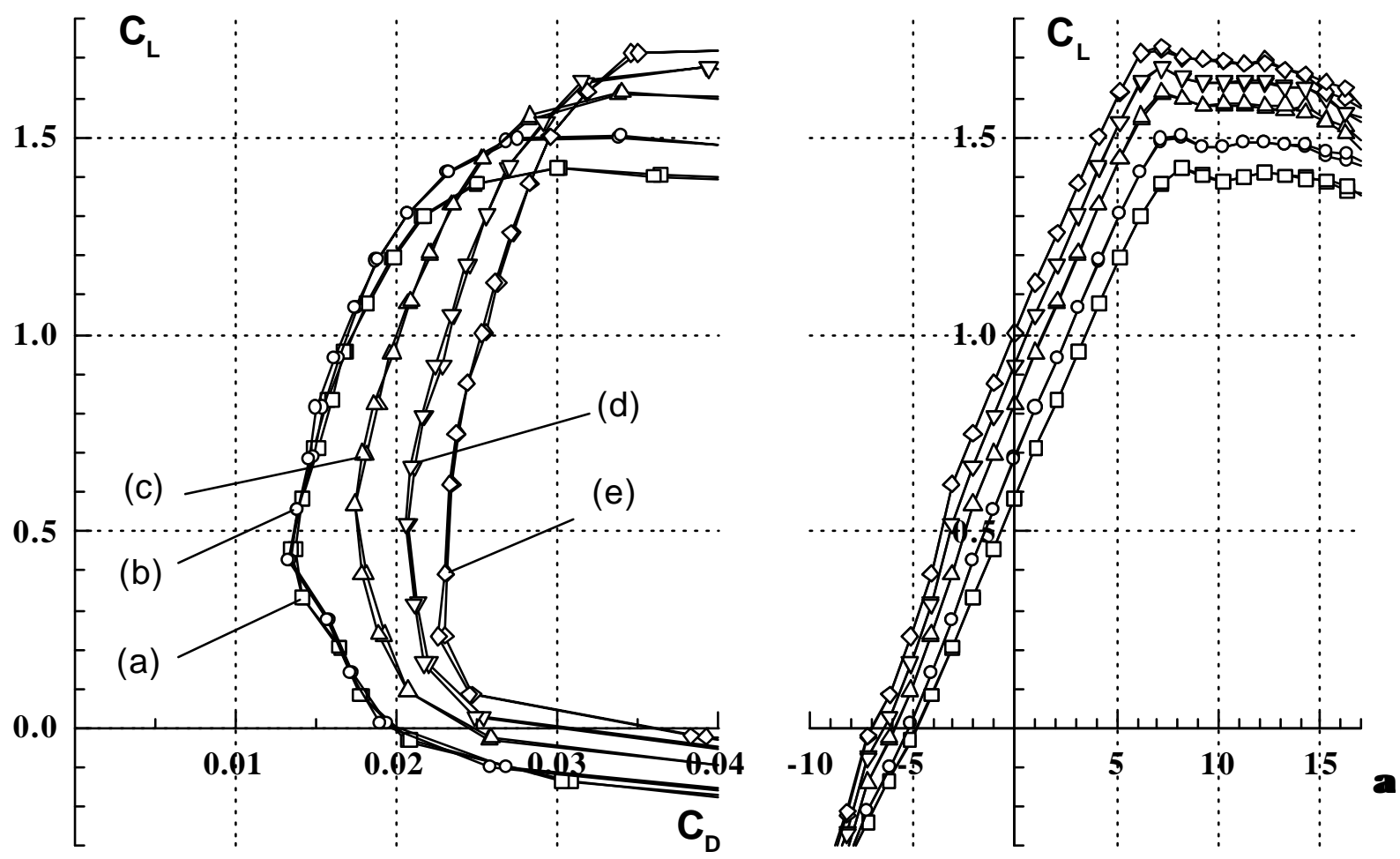

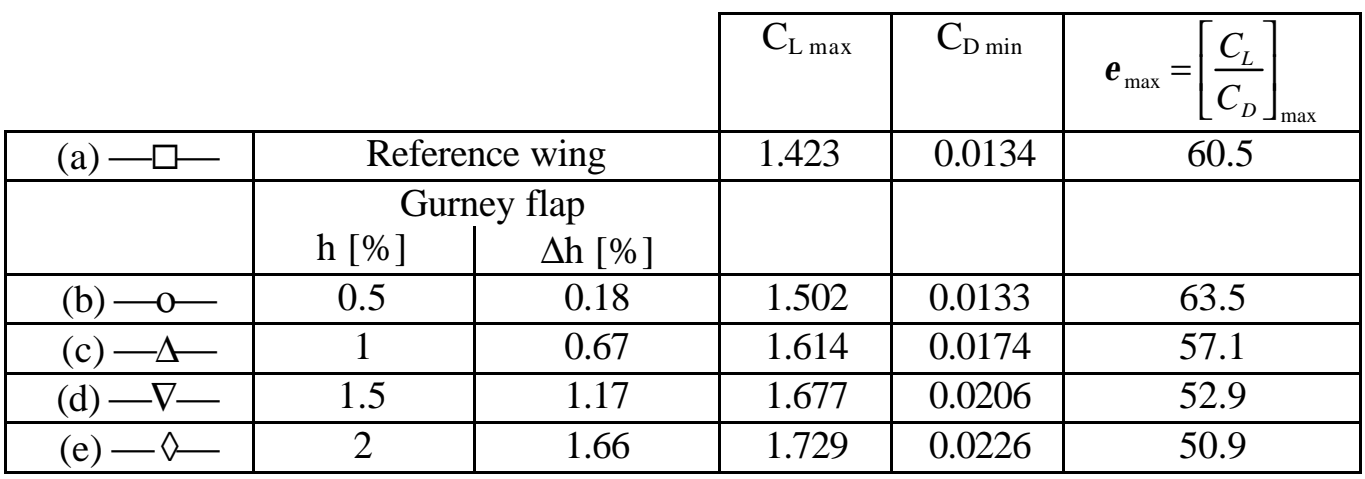

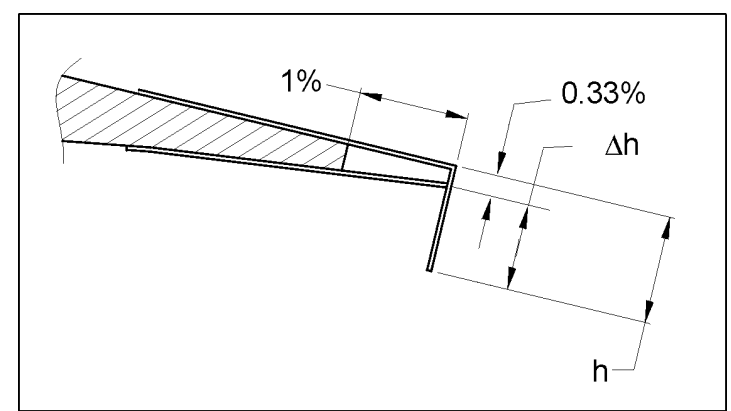

Fig. 6. Drag polars of the reference wing and of the same wing with various Gurney flap heights. Airfoil: HQ17, $R e=0.5 \times 10^{6}$. 


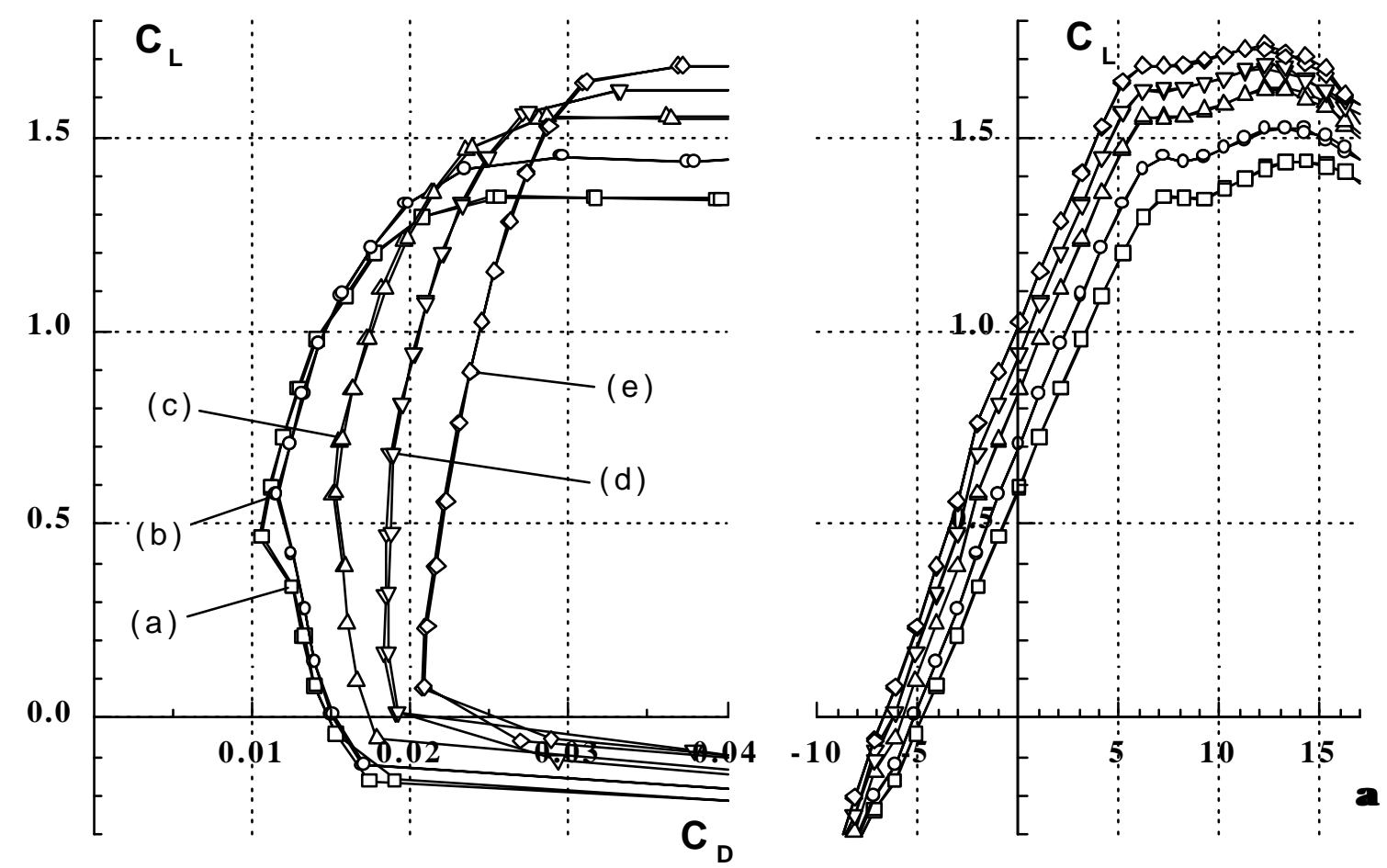

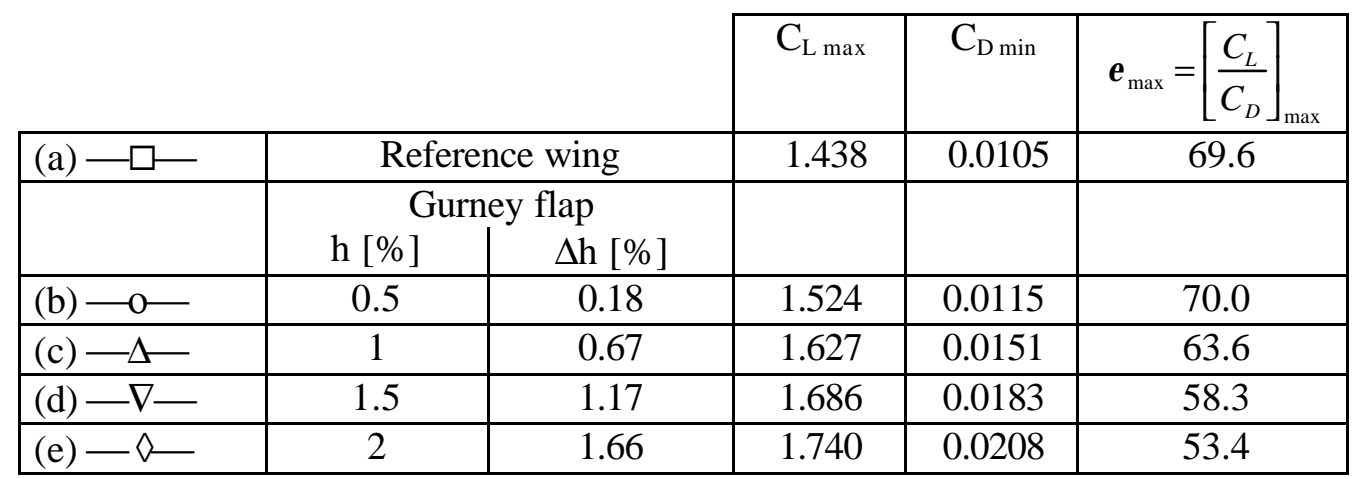

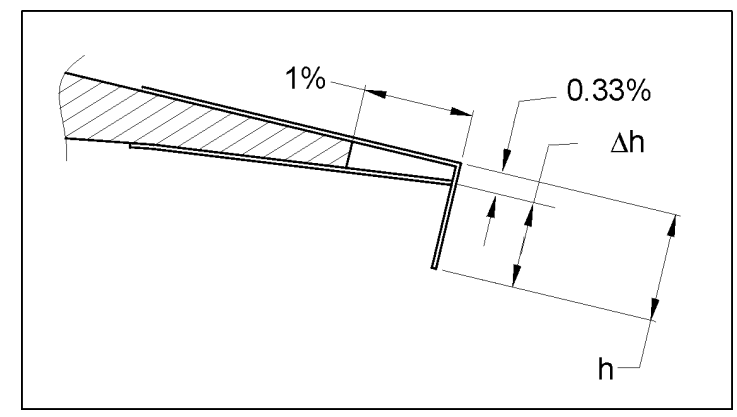

Fig. 7. Drag polars of the reference wing and of the same wing various Gurney flap heights. Airfoil: HQ17, $\operatorname{Re}=10^{6}$ 
We have also collected additional data with the Gurney flap attached to the trailing edge like in Fig. 1 (not shown in Figs. 6 and 7). It turned out that the relevant flap height $\Delta \mathrm{h}$ is in all cases the flap height which is added to the finite thickness of the trailing edge of the reference wing. Usually, we will normalise this effective flap height $\Delta \mathrm{h}$ with the chord length $\mathrm{c}$ of the airfoil, i.e., we will deal mostly with the expression $\Delta \mathrm{h} / \mathrm{c}$. Further, we will define the device drag coefficient $\Delta \mathrm{C}_{\mathrm{D}}$ as the difference between the minimum drag coefficient of the airfoil with Gurney flap and of that without, i.e., that of the reference wing. It turns out to be useful to normalise $\Delta \mathrm{C}_{\mathrm{D}}$ with the normalised flap height $\Delta \mathrm{h} / \mathrm{c}$. The dependence of drag increase and lift increase on $\Delta \mathrm{h} / \mathrm{c}$ can be seen in Fig. 8. In order to highlight the increase of lift due to the Gurney flap, we normalise the maximum lift with Gurney flap $\mathrm{C}_{\mathrm{Lmax}}$ by the maximum lift without Gurney flap $\mathrm{C}_{\mathrm{LOmax}}$. These normalised data can be also seen in Fig. 8. For comparison, we have also plotted the displacement thickness of the boundary layer $\delta$ on the lower side of the reference wing just at the trailing edge and for the angle of attack of lowest drag $\left(\alpha=-1^{\circ}\right)$.
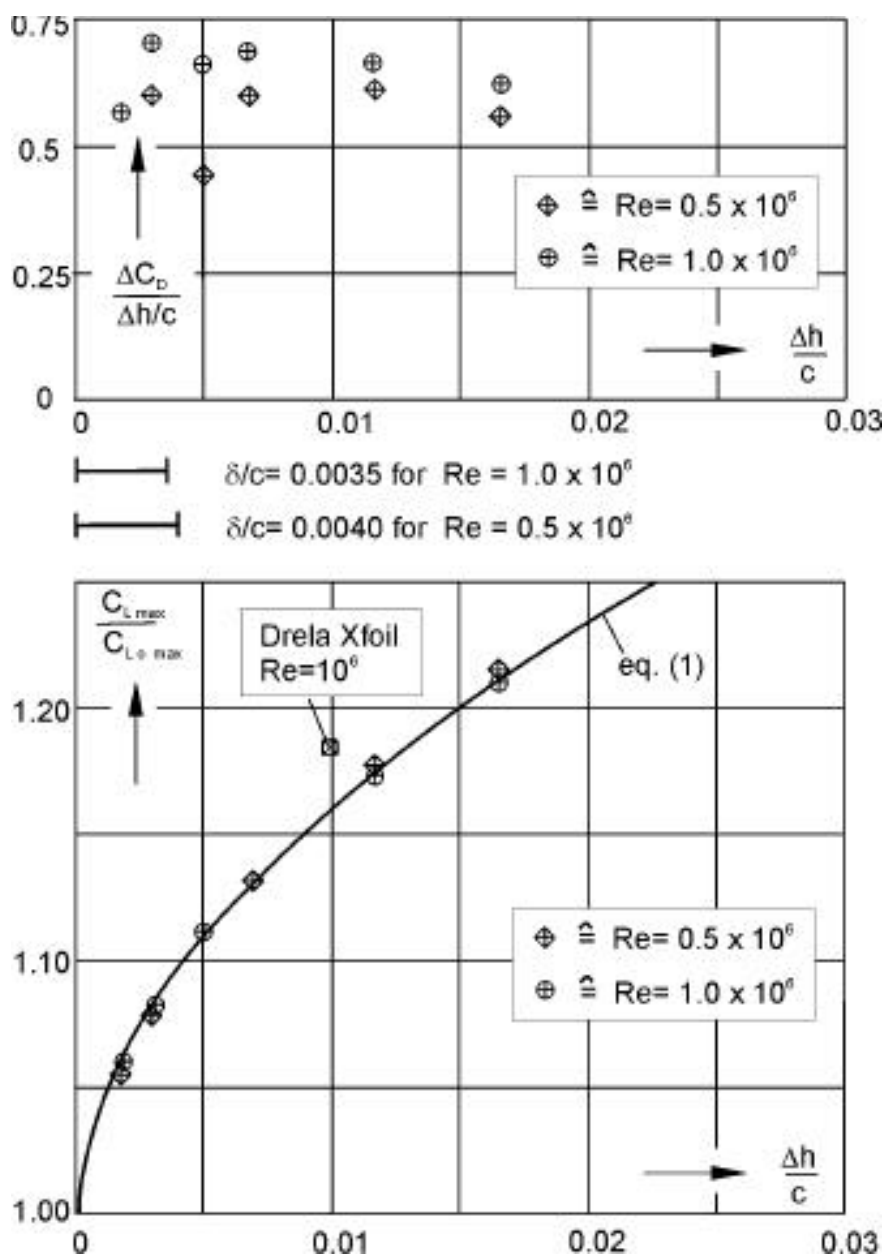

Fig. 8. The dependence of device drag and lift increase on Gurney flap height.

If the device drag $\Delta \mathrm{C}_{\mathrm{D}}$ is normalised with the normalised Gurney flap height $\Delta \mathrm{h} / \mathrm{c}$, one obtains an almost constant value of about 0.6. There are, however, slight differences between the data collected at the two Reynolds numbers. Much more surprising is the fact that the increase in lift does not show any difference between the two Reynolds numbers.

Of course, data collected at just two Reynolds numbers can be hardly generalised. Obviously, for significantly thicker boundary layers there should exist at least some deviation. Probably, with a thicker boundary layer, both the device drag and the lift increase are likely to be reduced. This is what we see if we replace the Gurney flap by a quarter circle and calculate theoretical data with the Drela code [14]. Possibly, in this particular application, this code may be already stretched beyond its validity range. 
In addition, a turbulator was not present in our calculation with this code. We know from previous experiments that, with a laminar separation bubble being present on the lower side of the airfoil, one obtains results differing from the ones being shown here.

Nevertheless, playing with the Drela code [14] confirms another hunch of the authors. For a potential flow solution where the flow can creep around the upper sharp edge of the Gurney flap, one obtains about double the lift increase than for the case with viscosity and boundary layer development taken into account. Roughly speaking, this means that given a sufficiently thin boundary layer on the lower side of the airfoil, this lower side behaves similar to the potential case in its ability to create a downwash downstream of the trailing edge. On the other hand, the flow from the upper side does not care and separates from the upper edge of the Gurney flap. Of course, this cannot be exactly correct, but it provides a feeling for what happens. And, of course, we are aware of the complexity of the real flow, as measured and calculated by Sauvage [5].

Returning to an interpretation of our own measured data: One may wonder whether or not it is possible to improve the lift to drag ratio $\varepsilon$ of an airfoil at low subsonic flow speeds with the help of a Gurney flap. In Fig. 9 we show data of $\varepsilon_{\text {max }}$, i.e., the best lift to drag ratio being achieved for various Gurney flap heights, normalised with the best lift to drag ratio $\varepsilon_{0 \max }$ of the reference wing. It appears as if at very low Gurney flap heights there may be a very small regime where an improvement could be possible. However, looking at the data in Fig. 9, one may argue that this could be a spurious effect as well. Nevertheless, the model calculations in the next section will shed much more light on this issue.

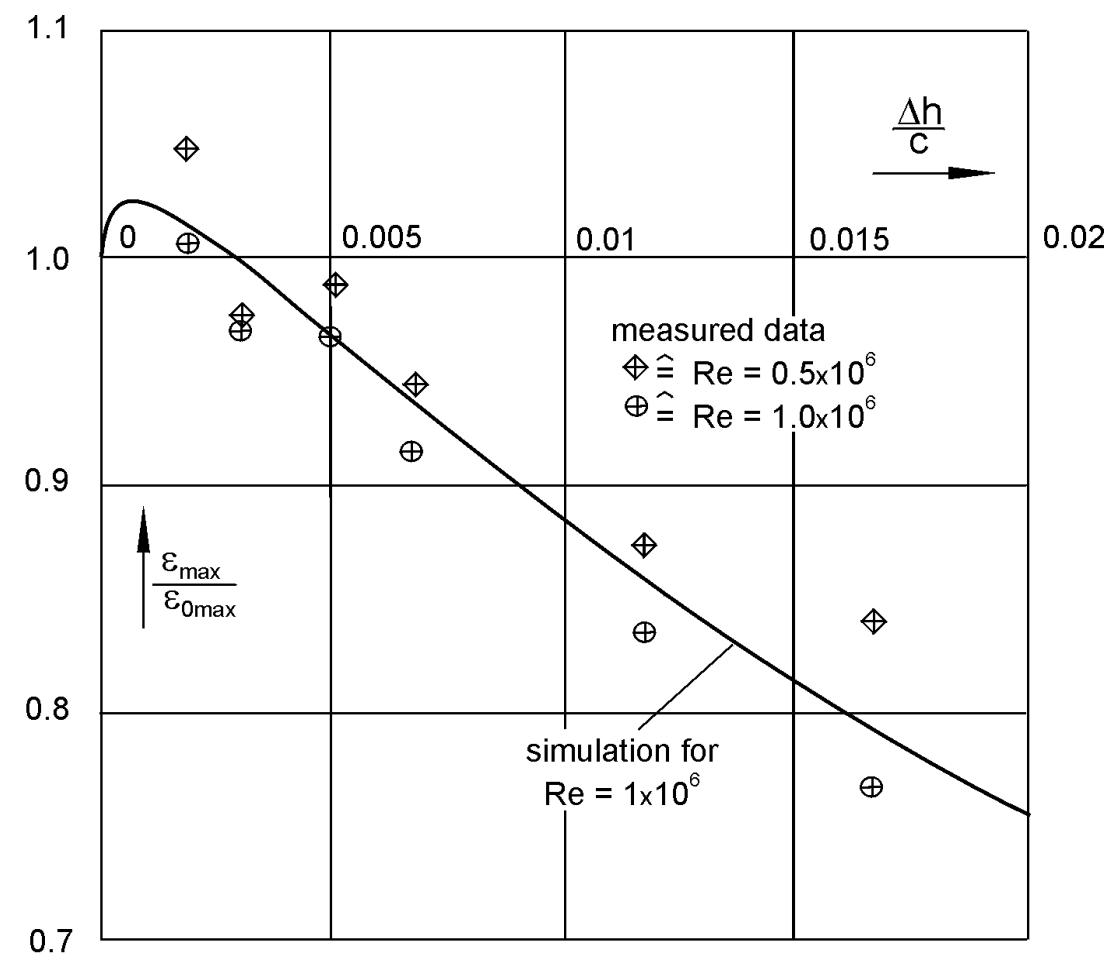

Fig. 9. Lift to drag ratio of various Gurney flap heights. 


\subsection{Simplified Simulation}

Basically, if the Gurney flap size is increased, the drag polar is shifted to the right hand side towards higher drag. At the same time, the drag polar is also shifted upwards, which results in a higher maximal lift. However, as Fig. 8 clearly shows, both dependences are different.

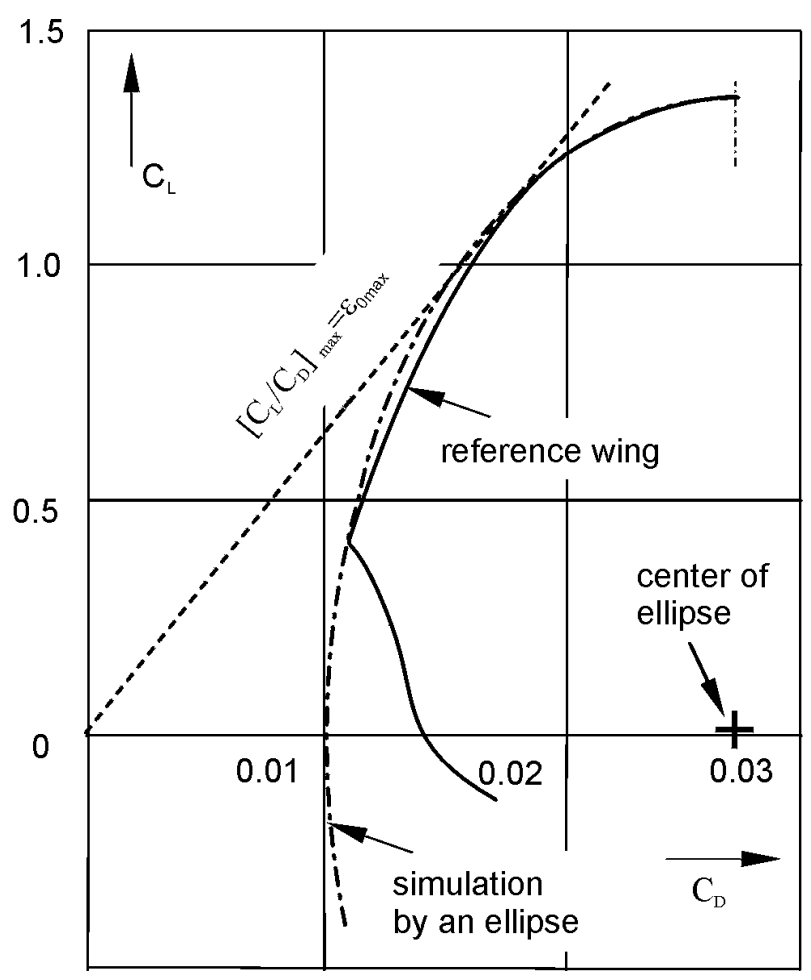

Fig. 10. Emulation of the drag polar by an ellipse.

As a highly simplified model ansatz, we approximate the drag polar by an ellipse, which emulates the shape in the relevant regime of the drag polar where the best lift to drag ratio $\varepsilon_{\max }$ is expected. In a second step, this ellipse is shifted to the right hand side by an approximation of the data in Fig. 8, i.e., by assuming that the device drag is $\Delta C_{D}=0.6^{*}(\Delta h / c)$. In a third step, the ellipse is shifted upwards to represent the increase in lift. The lift increase can also be simulated in a straightforward way by another empirical formula, which is an adjustment to the data in Fig. 8.

$$
C_{L \max } / C_{L 0 \max }=1+1.9661^{*}(\Delta h / c)^{0.5442}
$$

It can be seen in Fig. 8 that this latter approximation is excellent. Using these two empirical laws for the device drag and for the lift increase, one can calculate analytically what the best lift to drag ratio $\varepsilon_{\max }$ of the airfoil with Gurney flap is. Normalising $\varepsilon_{\max }$ with the best lift to drag ratio of the reference airfoil $\varepsilon_{\max 0}$, one obtains the curve in Fig. 9.

It is interesting to see that, indeed, there is a narrow regime at very small Gurney flap heights where the lift to drag ratio is improved above that of the reference wing. In addition, there is a perceivable increase of lift (8\%) at that $\Delta h / c$ where the $\varepsilon_{\max } / \varepsilon_{0 \max }$ curve cuts through the horizontal axis, i.e., at the same lift to drag ratio which is obtained by the reference wing. This means that $\left(\varepsilon_{\max } \cdot \sqrt{\mathrm{C}_{\mathrm{L}}}\right)^{-1}$, an expression which governs the sinking rate of an aircraft, is improved. 


\subsubsection{Lift-independent additional drag}

Obviously, also other contributions to the total drag of an aircraft exist besides the drag of the airfoil alone. Assuming that this additional drag is not dependent on the lift (a critical assumption, as we will see), it will only cause a shift of the drag polar to the right hand side. For increasing total drag, the device drag of the Gurney flap becomes less important. Thus, the increased lift due to the Gurney flap produces a better lift to drag ratio. We have calculated a number of cases where we have assumed various levels of additional drag. In Fig. 11 a fan of curves can be seen. We have adjusted the level of the additional drag in a manner so that the reference wing has a lift to drag ratio $\varepsilon_{0 \max }$ of $20 ; 30 ; 40 ; 50$. In addition, the curve for the reference wing without additional drag $\left(\varepsilon_{0 \max }=65\right)$ is shown. The curves in Fig. 11 are then calculated using the procedure which had been described in the preceding section.

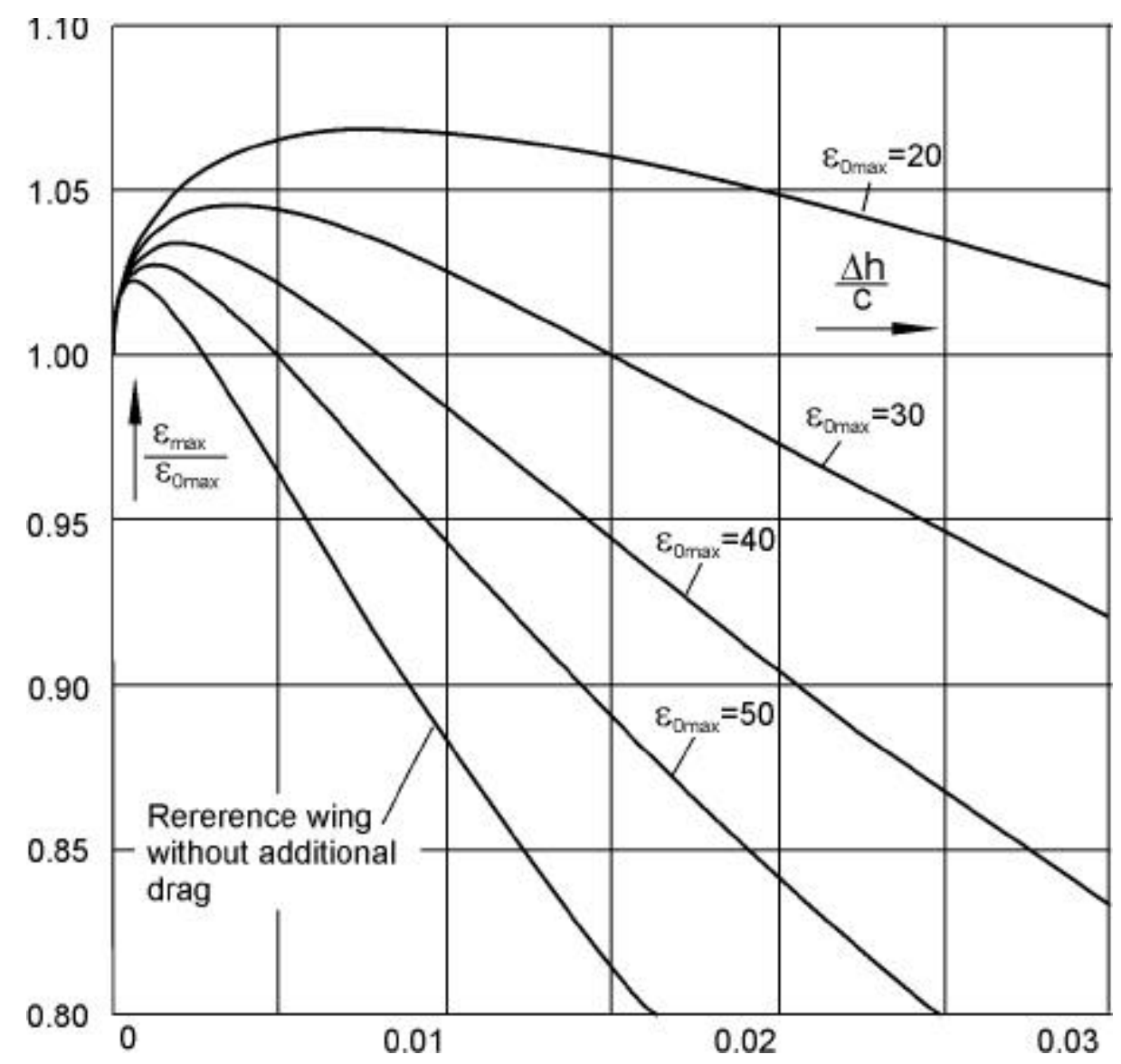

Fig. 11. Lift to drag ratio $\varepsilon_{\max }$ of a wing with a Gurney flap. It is assumed that additional drag besides the airfoil drag exists, which is not dependent on the lift. The additional drag is adjusted so that the reference wing (without Gurney flap) develops a lift to drag ratio $\varepsilon_{0 \max }$ of $20 ; 30 ; 40 ; 50$. The value of $\left(\varepsilon_{0 \text { max }}=65\right)$ refers to the reference wing without additional drag. 


\subsubsection{Lift-dependent additional drag}

Fig. 11 is, however, deceiving as to what can be achieved with a Gurney flap. It turns out that it is necessary to take also lift-dependent drag into account. A typical contribution of this type of drag is the induced drag of a wing due to its finite aspect ratio $\Lambda$. As another (related) example, lift dependent drag in a turbomachine can come from tip vortices of the blades. For a wing with an elliptical lift distribution, the induced drag is

$$
C_{D i}=\frac{C_{L}^{2}}{\pi * \Lambda}
$$

Taking this induced drag into account as the only additional drag contribution yields quite disappointing results, see Fig. 12. Actually, under these circumstances, practically no regime exists anymore where the Gurney flap would offer improvements. This is basically because the induced drag law of equation (2) shifts the best lift to drag ratio $\varepsilon_{\max }$ to comparatively low lift coefficients where a Gurney flap does not provide any advantage. In other words: At high lift coefficients where the Gurney flap would be advantageous, there is an enhanced drag penalty connected with that high lift coefficient, see equation (2).

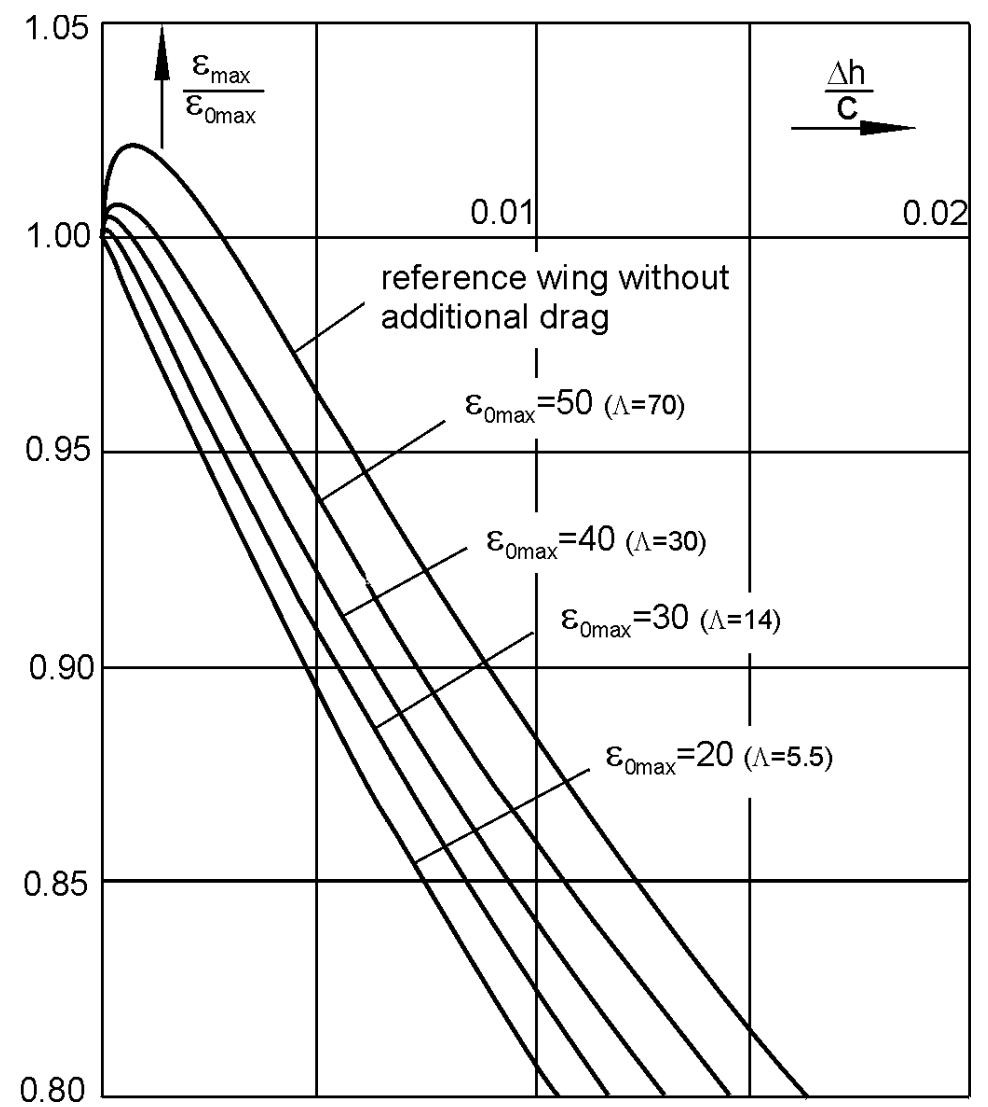

Fig. 12. Gurney flap performance for a wing with additional induced drag. The levels of the induced drag were set by a suitable choice of the wing aspect ratio $\Lambda$ so that lift to drag ratios of the reference wing were adjusted to $\varepsilon_{0 \max }$ of $20 ; 30 ; 40 ; 50$ 


\subsubsection{Combined additional drag: lift-independent and lift-dependent}

With both contributions, lift-independent and lift-dependent drag being present, the situation looks less gloomy. In order to demonstrate that, we provide in Fig. 13 results for two basic lift to drag ratios of the reference wing, i.e., $\varepsilon_{0 \max }=20$ and $\varepsilon_{0 \max }=30$. These values are typical for commercial aircraft and turbomachine applications.
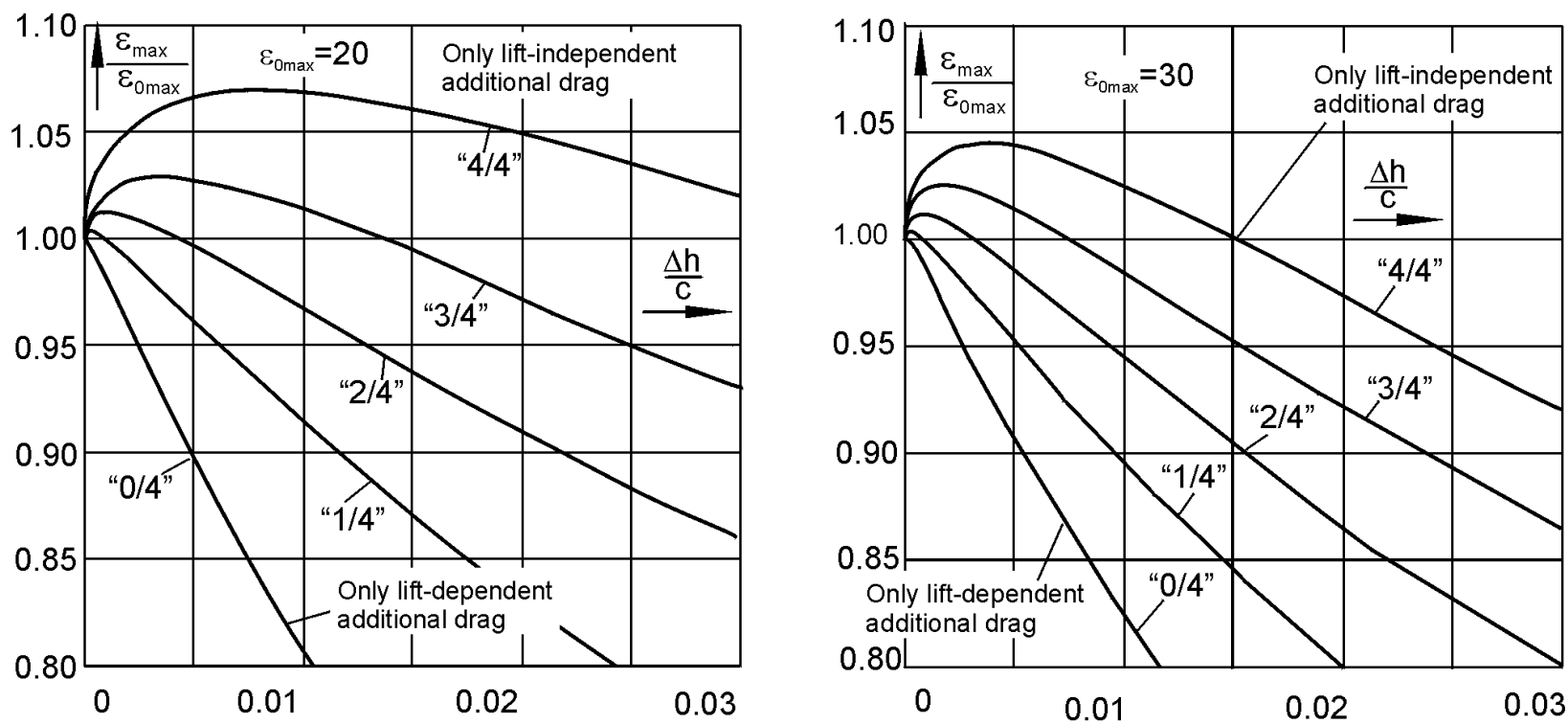

Fig. 13. Data of a wing with Gurney flap and additional drag consisting of lift-independent drag and liftdependent drag. Four cases are considered: Only lift-independent drag exists for the " $4 / 4$ " case. For the " $3 / 4$ " case this contribution is reduced to $75 \%$ of the " $4 / 4$ " case. The rest of the drag is adjusted with a finite wing span to obtain $\varepsilon_{0 \max }=20$ or 30 , respectively. The procedure is analogous for the " $2 / 4$ " and " $1 / 4$ " cases. The " 0 " case has only induced drag as additional drag.

As can be seen in Fig. 13, one can obtain a beneficial effect of the Gurney flap if the share of the induced drag is not too significant.

Surprising, however, is the fact that the general drag characteristics of the wing are of such a crucial importance.

We do not claim that the above model calculations will provide accurate predictions in all situations. However, we have also collected data with a swept wing of a modern commercial aircraft with an airfoil with high rear loading. These data are compatible with the above considerations.

In our calculations, the curves in Fig. 11, are analytical results from a closed form solution. However, taking also the induced drag into account does not permit a closed form solution anymore but requires an (albeit simple) iteration process to determine $\varepsilon_{\max }$. Of course, alternatively, everything can also be done with a look-up table of the real measured data points and with a simple and purely numerical iteration process. Anyway, the above model calculations provide a useful insight into a sensible parameter selection for Gurney flaps. Typically, flap heights of $0.3-0.5 \%$ will be chosen for a cruise flight situation, providing an increase of lift of about $7-12 \%$ for single airfoils.

Finally, it should be mentioned that Gurney flaps are also very well suited for high-lift multi-element wings. Because there the lift coefficients are already very high, the lift increases being obtained are lower, if counted in percent. Nevertheless, it is a well established fact that a Gurney flap still enhances the lift on top of an already high-lift yielding multi-element wing. 


\subsection{Other shapes of mini-flaps}

Obviously, there is a small separation regime which starts just upstream of the Gurney flap on the lower side of the airfoil. One may wonder what the device drag reduction is if this corner regime is filled with model clay so that a quarter of a circle is formed. As Fig. 15 shows, there is an improvement, but it is marginal. We observe a very slight decrease of the drag and an equally slight increase in lift.

On the other hand, a diverging trailing edge behaves rather differently, see Fig. 14. The drag is considerably decreased but the lift is also decreased, if the diverging trailing edge is compared with a Gurney flap of identical height $(\mathrm{h}=1 \%)$. Actually a smaller Gurney flap with $\mathrm{h}=0.82 \%$ (or slightly less) produces very similar results. Thus, a diverging trailing edge works indeed very similar to a Gurney flap, but a smaller Gurney flap produces already the same effect as a bigger diverging trailing edge. On the other hand, small is not always beautiful: The greater height of the diverging trailing edge provides a greater mechanical stiffness and stability of the wing, which can be translated into a lower weight of the wing, which is certainly desirable.

In our paper we will, however, stick to the simple Gurney flap shape. All devices for drag reduction which we develop in the following sections will be, nevertheless, also valid for diverging trailing edges.

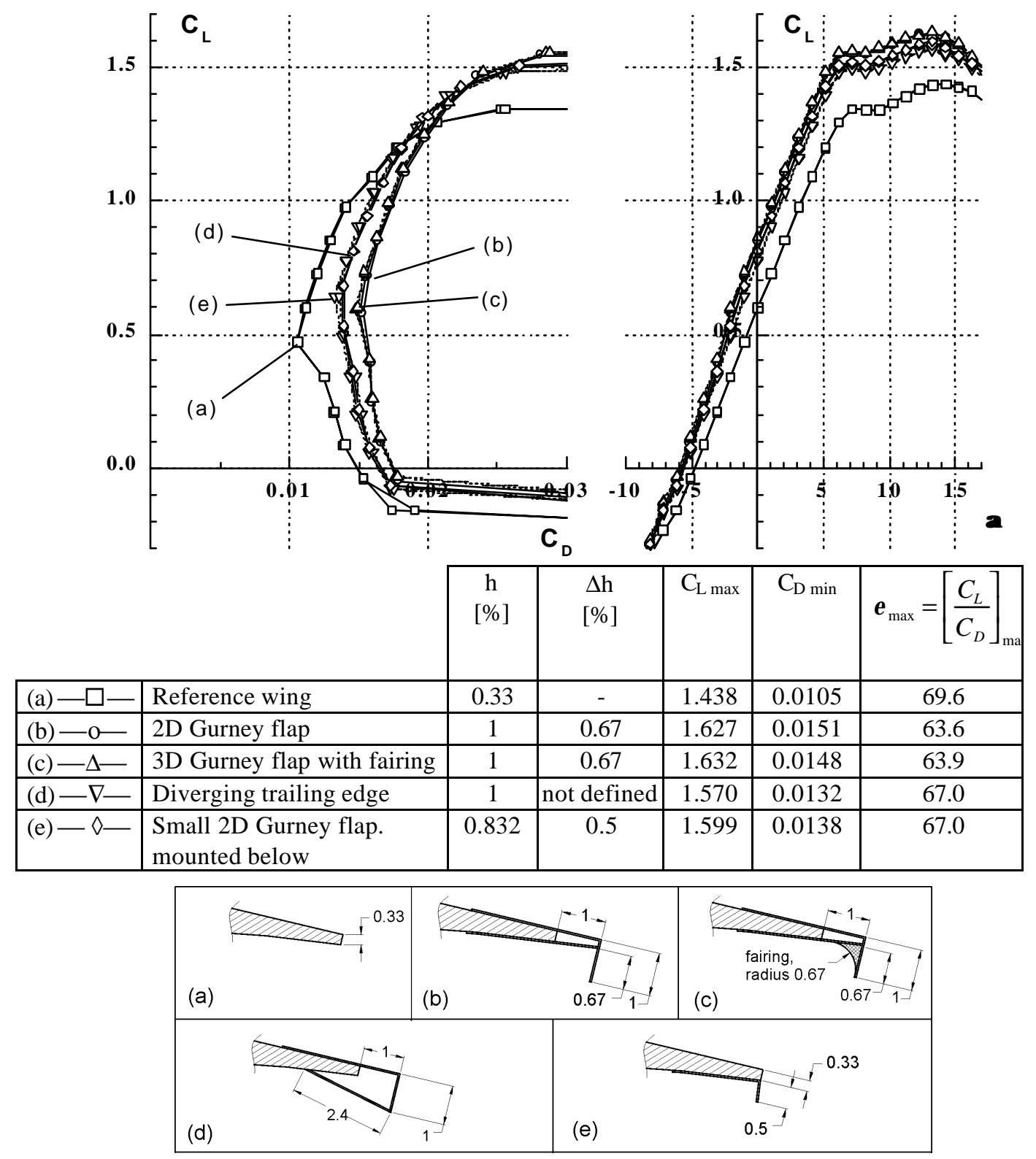

Fig. 14. Comparison of a diverging trailing edge with a Gurney flap and the influence of avoiding flow separation in front of a Gurney flap by fairing. 


\section{Drag reduction by wake stabilisation.}

\subsection{Concept, test arrangement and proof of absolute instability}

In the separated flow downstream of a Gurney flap we expect that an absolute instability [15-17] can be observed. One well-known example of an absolute instability is the Karman vortex street which occurs in the wake of a cylinder. Such instabilities occur not only in laminar flows, as the classical experiments with cylinders would suggest, but they can be also observed in turbulent flows. A tell-tale sign for an absolute instability is the occurrence of a single peak in the spectrum of the fluctuations in or near the wake. The reversed flow in the wake is a property which enhances this instability [17]. Since trailing edges of airfoils are seldomly very sharp, we also expect such an absolute instability in the wake of an (even slightly) blunt trailing edge.

Our first aim is, therefore, to demonstrate experimentally, that a single frequency does exist in the fluctuations downstream of the trailing edge, and then devise trailing edge modifications to suppress those periodic fluctuations.

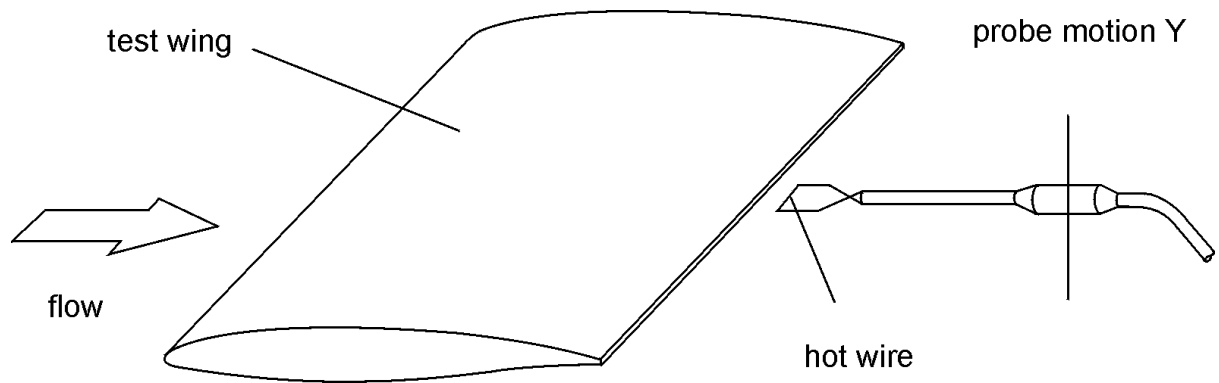

Fig. 15. Test arrangement for wake measurements

The easiest way to measure velocity fluctuations is offered by the well-established hot wire anemometry. The hot wire instrument which we have used was a DLR-HDA IIIf anemometer with a built-in analogue linearizer. The anemometer output signals were plotted directly with an X-Y-plotter in order to obtain the mean flow distribution downstream of the wing. In places of maximum mean velocity gradient the highest streamwise velocity fluctuations u' occurred. The u' fluctuation signals were Fourier-analysed and the spectra were plotted. Fig. 16 shows recorded data of the reference wing. Due to the finite thickness of the trailing edge of this wing, there is a small hump in the spectrum, signifying an (albeit weak) absolute instability, which is highlighted by its single resonance frequency $f=2.4 \mathrm{kHz}$. By the way, this hump vanishes for a very sharp trailing edge.

As Fig. 17 shows, the wake of a (2-D) Gurney flap is wider, indicating a higher drag. In addition, the u'-fluctuation levels are higher and the periodic constituent with the frequency $\varnothing=0.8 \mathrm{kHz}$ is much stronger. The data are recorded at $\operatorname{Re}=1 \times 10^{6}$. Similar data are obtained if the mean flow velocity is reduced to one half of its previous value, yielding a Reynolds number of $0.5 \times 10^{6}$. As expected, the peak frequency $f_{0}$ is also reduced to half of its previous value. If one calculates a Strouhal number with the frequency $f_{0}$, the Gurney flap height ${ }^{1}$ and the mean flow velocity, one obtains a resonance Strouhal number of $S \approx 0.14$.

\footnotetext{
${ }^{1}$ It is conceivable that other reference lengths like, e.g., the momentum loss boundary layer (or wake) thickness may be a more appropriate reference length here.
} 

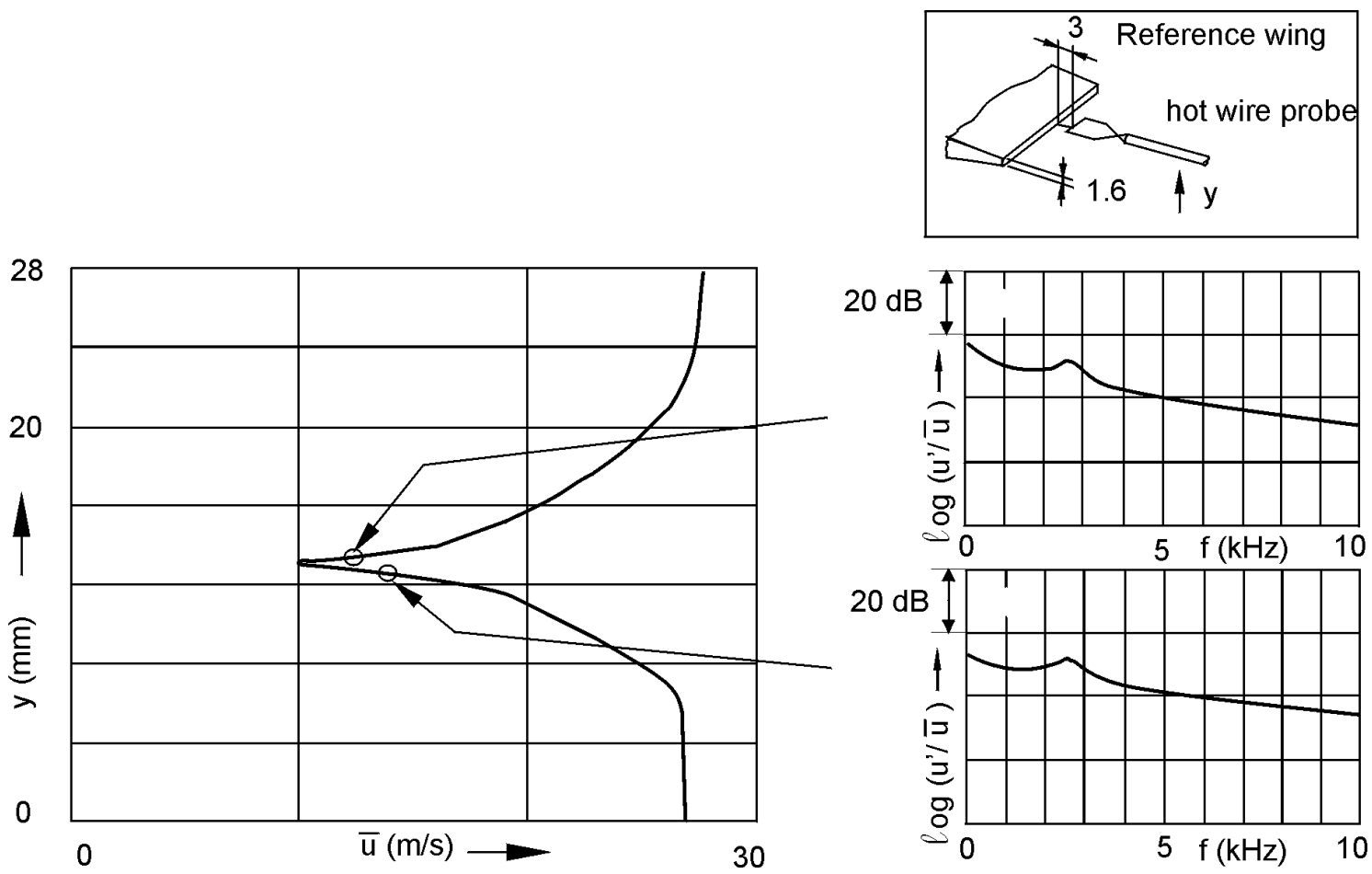

Fig. 16. Reference wing with finite trailing edge thickness of $0.33 \%$ chord. Data collected at a distance 3 $\mathrm{mm}(0.6 \%$ chord $)$ downstream of the trailing edge. Mean flow $\bar{u}$ distribution and $u^{\prime}$ fluctuation spectra were collected at two locations with steep mean flow velocity gradient. Airfoil HQ17, $\operatorname{Re}=1 \times 10^{6}, \alpha=-1^{\circ}=$ Angle with minimal drag, $C_{D}=0.0105 ; C_{L}=0.42$. Dimensions in $\mathrm{mm}$.
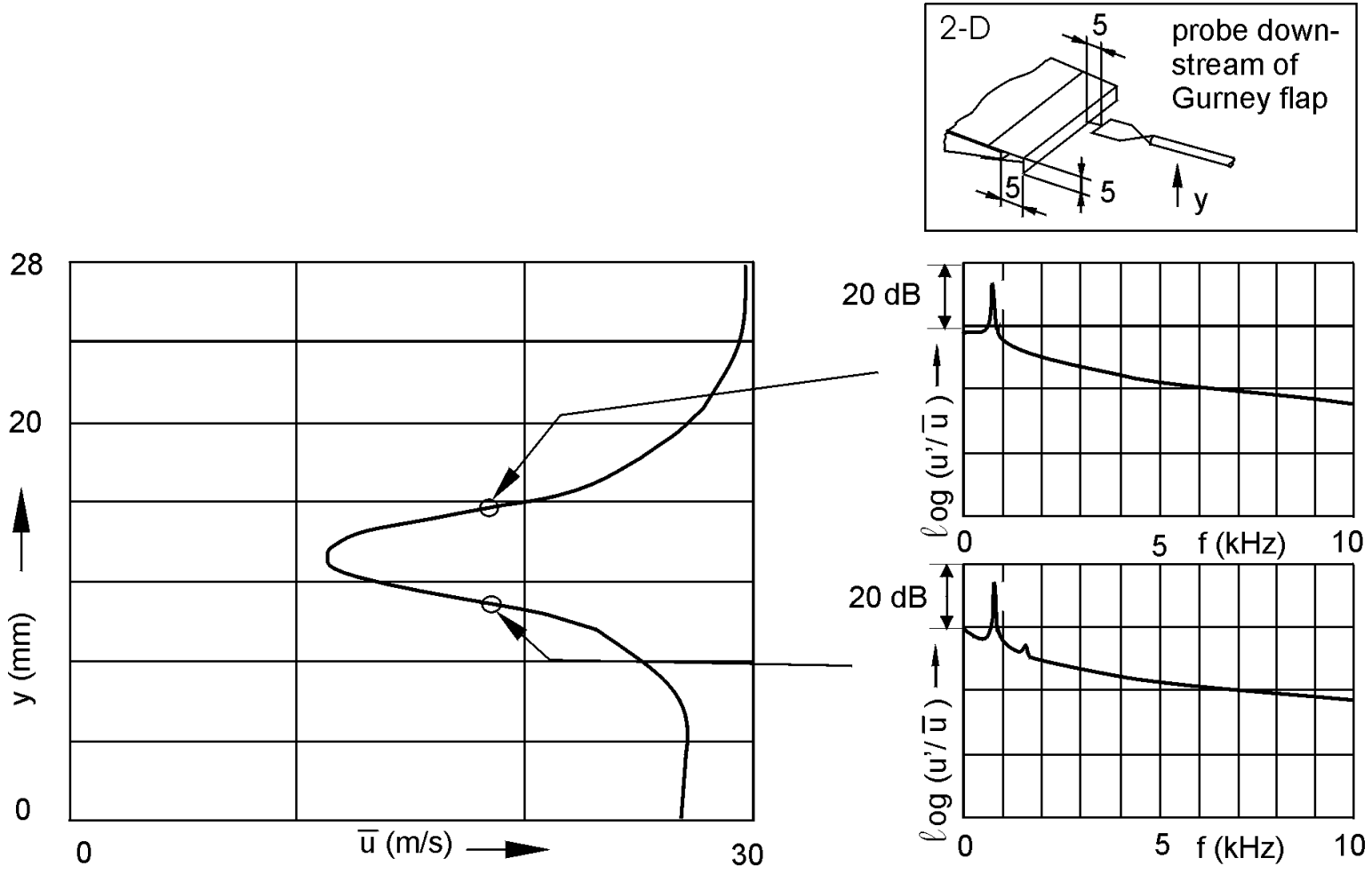

Fig. 17. Flow distribution and spectra in the wake of a 2-D Gurney flap with a height of $1 \%$ chord. Data collected at a distance of $5 \mathrm{~mm}(1 \%$ chord) of the trailing edge of the Gurney flap. Reynolds number and angle of attack as in Fig. 16. The Gurney flap height which we use throughout our instability investigations has a total height of $5 \mathrm{~mm}(1 \%$ chord) with an effective height of $3.4 \mathrm{~mm}$ (0.67\% chord). 


\subsection{Gurney flaps with slits}

It is known from the Karman vortex street on cylinders, that a three-dimensional structure of the wake flow field effectively suppresses the absolute instability. On a cylinder, that can be achieved by applying a helical structure on its surface (i.e., the Screwton spiral) which can be seen quite often on industrial chimneys.

Obviously, the same approach cannot be literally transferred to our problem. However, as it will turn out, there are various possibilities to obtain a three-dimensional wake flow field. The one which we are considering first is the application of slits in the Gurney flaps. The effect of slits on the wake can be seen in Fig. 19. The hot wire data clearly show that the absolute wake instability (i.e., the peak in the spectrum) has almost completely vanished. Drag polars can be seen in Fig. 18. It is obvious that the device drag is considerably decreased, by $28 \%$. On the other hand, the bleed air through the slits causes the Gurney flap to appear smaller, which slightly decreases the gain in lift.
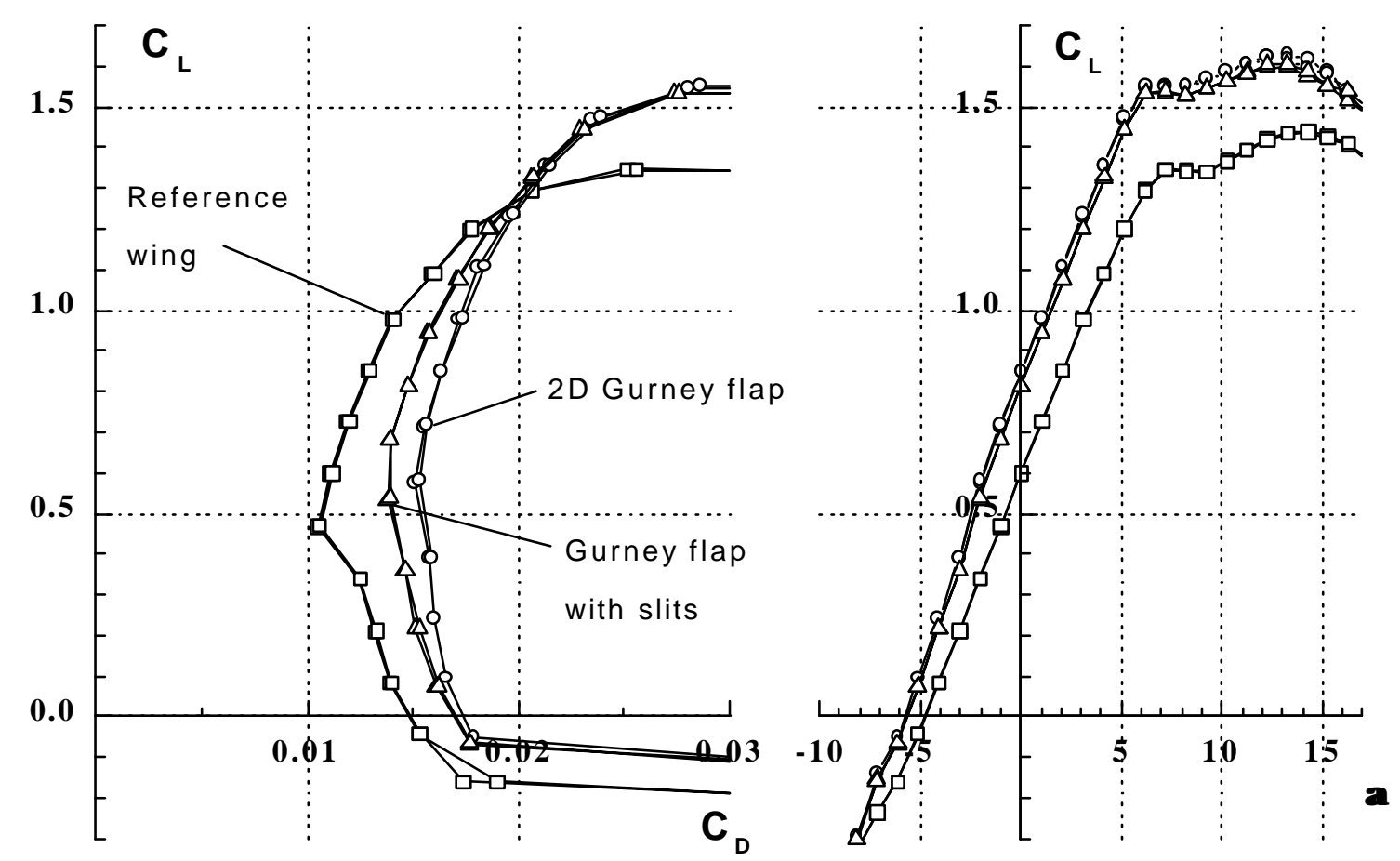

\begin{tabular}{|l|l|c|c|c|l|c|}
\cline { 3 - 7 } \multicolumn{2}{l|}{} & $\begin{array}{c}\mathrm{h} \\
{[\%]}\end{array}$ & $\begin{array}{c}\Delta \mathrm{h} \\
{[\%]}\end{array}$ & $\mathrm{C}_{\mathrm{L} \max }$ & $\mathrm{C}_{\mathrm{D} \min }$ & $\varepsilon_{\max }=\left[\frac{C_{L}}{C_{D}}\right]_{\max }$ \\
\hline$-\square-$ & Reference wing & 0.33 & - & 1.438 & 0.0105 & 69.6 \\
\hline$-0-$ & 2D Gurney flap & 1 & 0.67 & 1.627 & 0.0151 & 63.6 \\
\hline$-\Delta-$ & Gurney flap with slits & 1 & 0.67 & 1.605 & 0.0138 & 64.6 \\
\hline
\end{tabular}

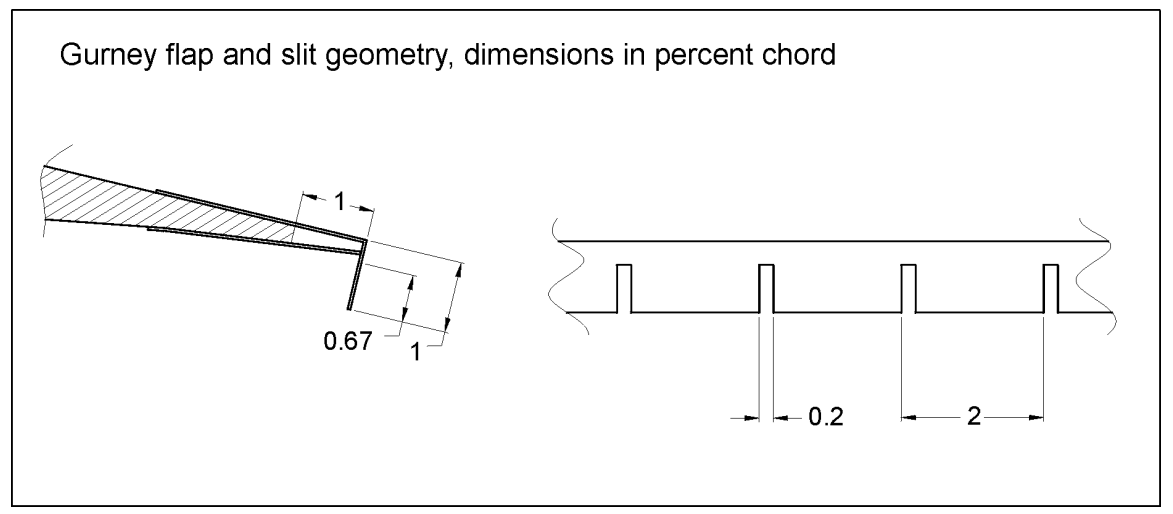

Fig. 18. Drag polars of a Gurney flap with slits. $R e=1 \times 10^{6}$ 
(a)
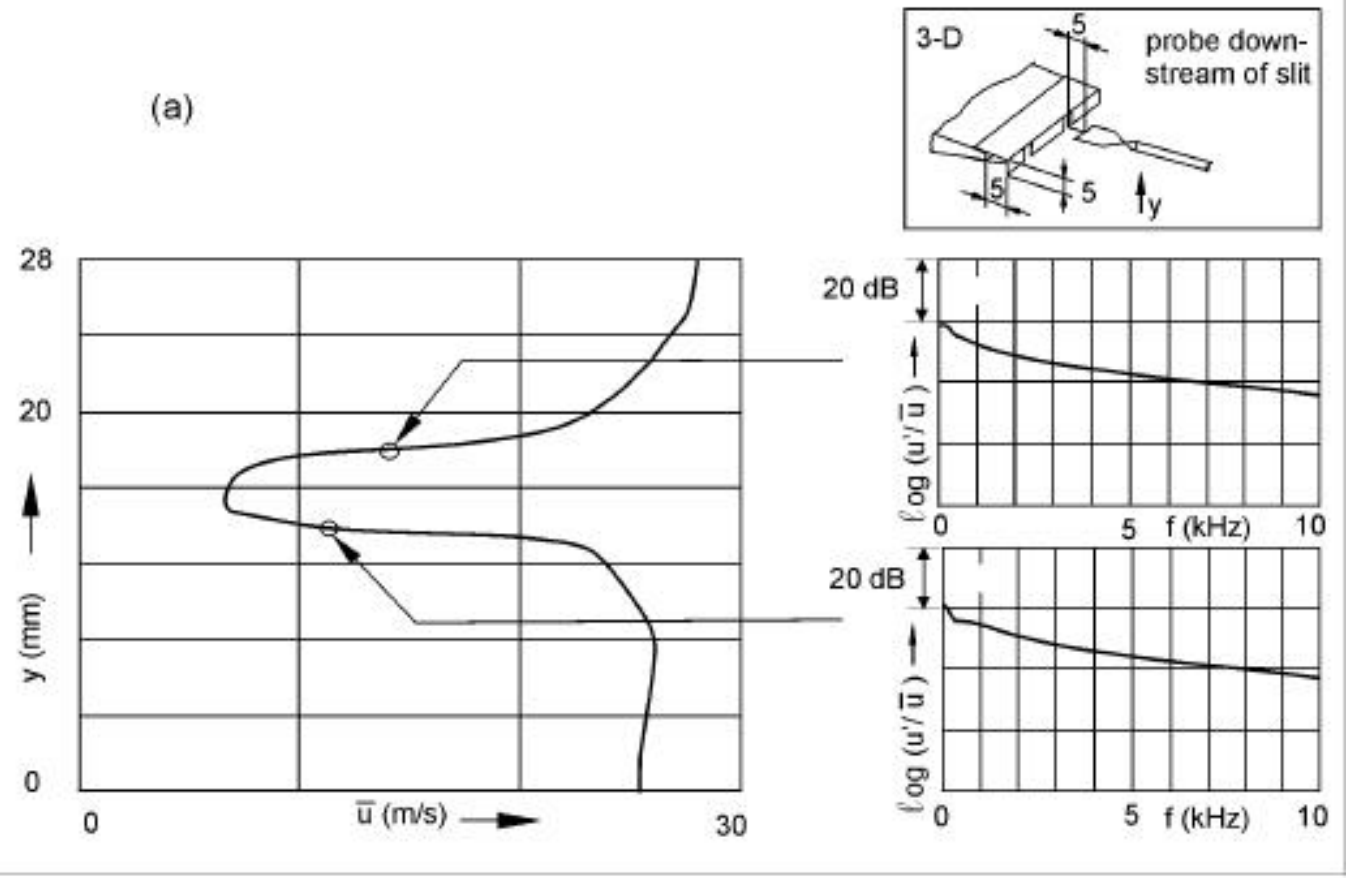

(b)
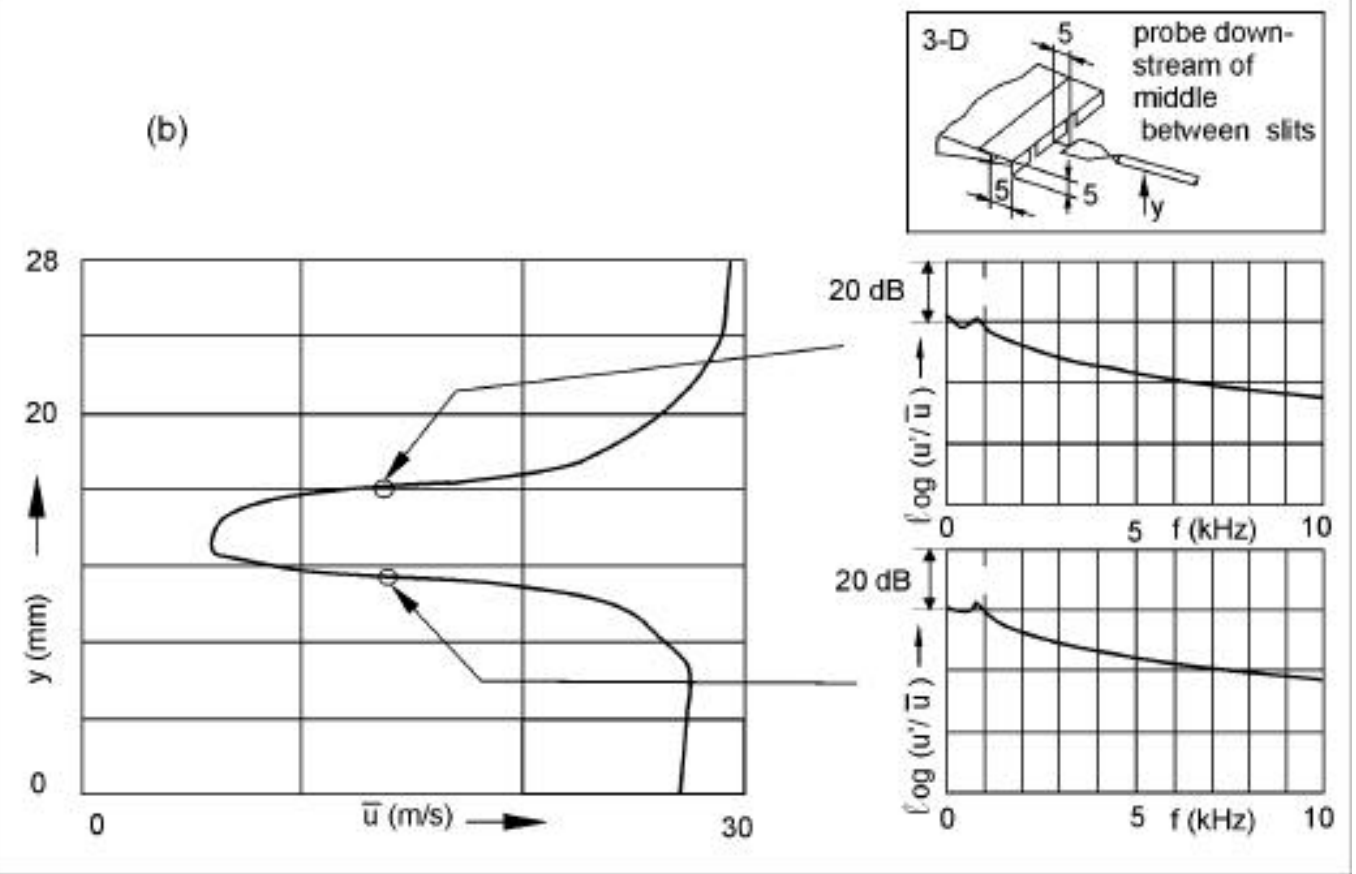

Fig. 19. Mean velocity distribution and spectra at $5 \mathrm{~mm}$ distance (1\% chord) downstream of a 3DGurney flap (height 1\% chord) with slits. Slit dimensions as shown in Fig. 18. Airfoil HQ17, $\operatorname{Re}=1 \times 10^{6}, \alpha=-1^{\circ}$. Upper diagram downstream of slit (a), lower diagram downstream of middle between slits $(b)$.

At higher angles of attack, however, the improvement due to the slits in the flaps in less pronounced. The hot wire data of the 2-D Gurney flap (not shown here) do not exhibit any resonance anymore either. As a matter of fact, Koch [16] has already predicted that the absolute instability disappears for strongly asymmetric wakes. Obviously, for high angles of attack, the wake of an airfoil becomes strongly asymmetric. Therefore, one cannot reduce anymore the drag at high angles of attack by instability suppression in the wake. On the other hand, drag reduction is particularly desired at low angles of attack, and, as Fig. 18 shows, it is indeed achieved there.

Finally, we expect that, due to the elimination of the absolute instability of the wake, also induced mechanical vibrations of the wing and radiated noise are very likely to be reduced. 


\subsection{Gurney flaps with holes}

One disadvantage of having slits in the Gurney flap is that the additional mechanical stiffness of the trailing edge with Gurney flap is no more available. Based on the idea that the reversed flow in the wake [5] is of crucial importance for the existence of an absolute instability [17] we considered holes in the Gurney flap as an alternative. This has the advantage that the mechanical stiffness and stability of the Gurney flap is maintained.

We have carried out tests with two sizes of holes, $0.3 \%$ chord and $0.5 \%$ chord in diameter. As Fig 20 shows, both are effective. The smaller holes draw less bleed air and thus cause lower losses in lift. By the way, the same data as with the smaller holes are obtained if the bigger holes are placed at double the lateral spacing. According to our hot wire data (not shown here), the elimination of the wake instability is not so complete as with the slits, but it is significant enough to cause an equivalent drag reduction.
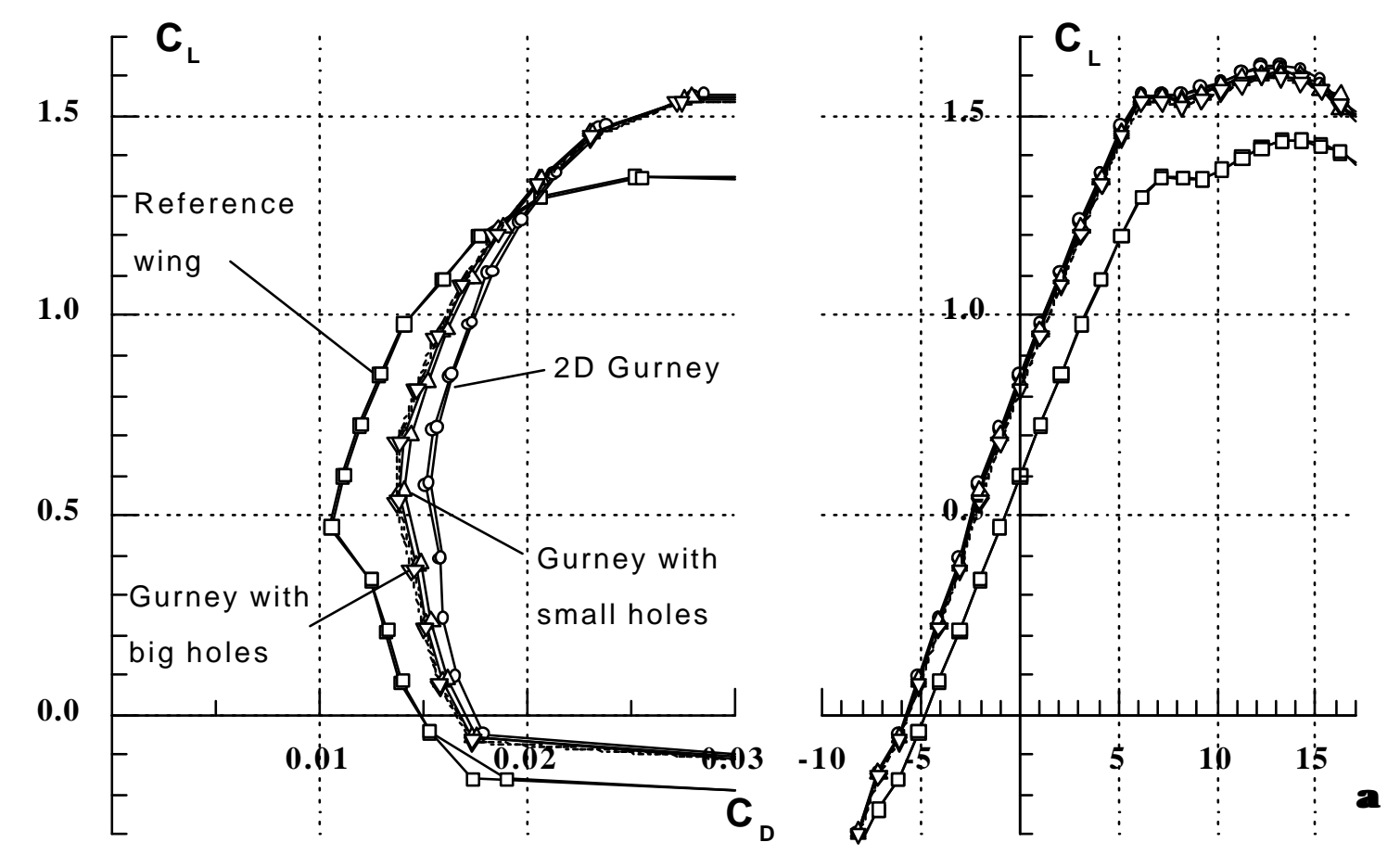

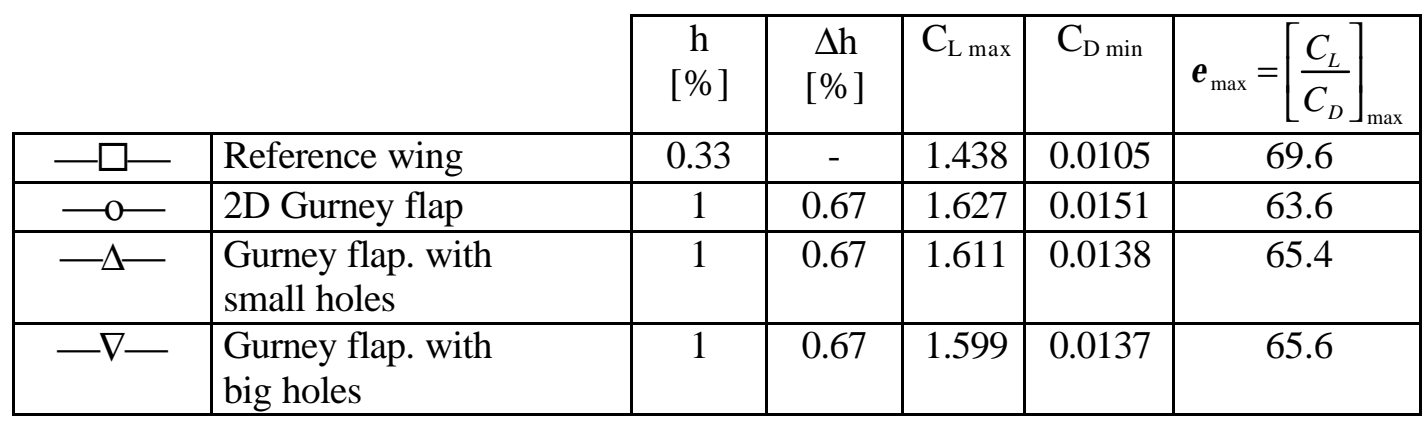

Gurney flap, hole geometry, dimensions in percent chord

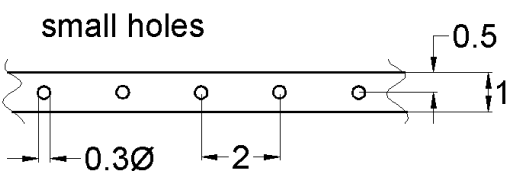

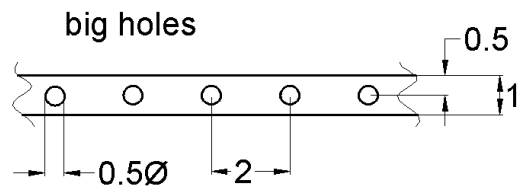

Fig. 20. Drag reduction by holes in the Gurney flap . 


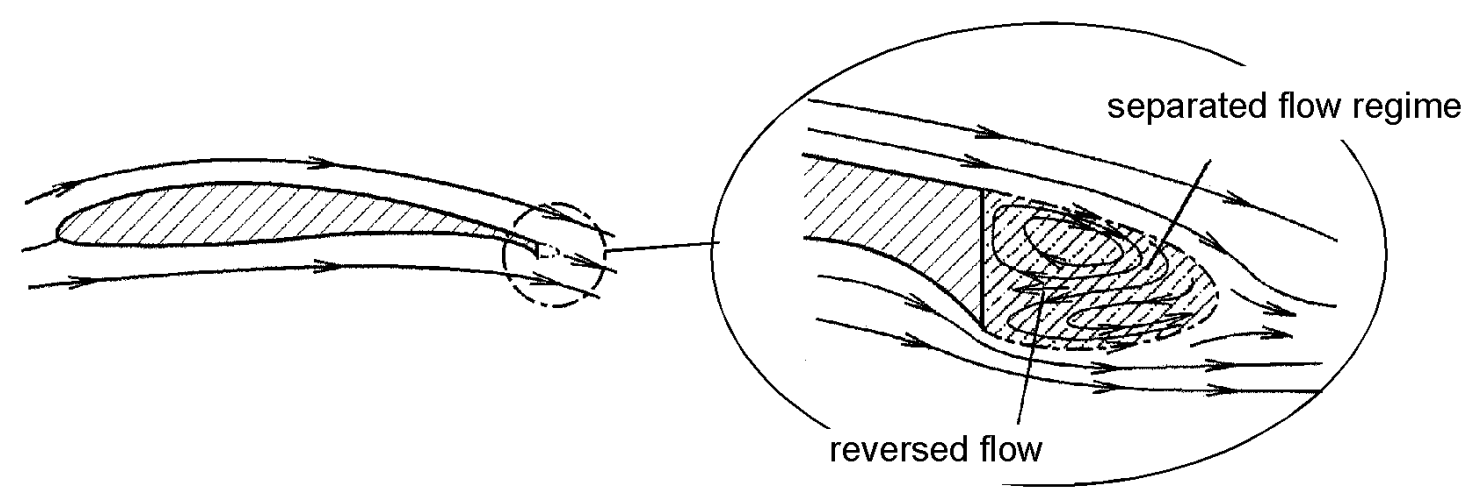

Fig. 21. Mean flow pattern in the wake downstream of a Gurney flap [5].

Fig. 21 shows a sketch of the mean flow pattern in the wake according to Sauvage [5]. In the middle of the regime of separated flow, a flow reversal occurs. From theoretical investigations by Huerre [17], it is evident that a shear flow with a flow reversal is prone to exhibit an absolute instability.

Thus, a simple idea is to prevent the flow reversal from occurring by obstacles in the wake. This can be achieved by vertical blades in the flow separation regime. It is, however, important to choose the configuration in a way that these inserted blades do not protrude out of the separation regime. Any perturbation of the high-speed mean flow outside the separation regime would cause losses and drag. The actual configuration of the wake stabilizers can be seen in Fig. 22. The vertical blades are attached to the Gurney flap by small non-obstructing supports with a spacing of $5 \mathrm{~cm}$ in the spanwise direction.

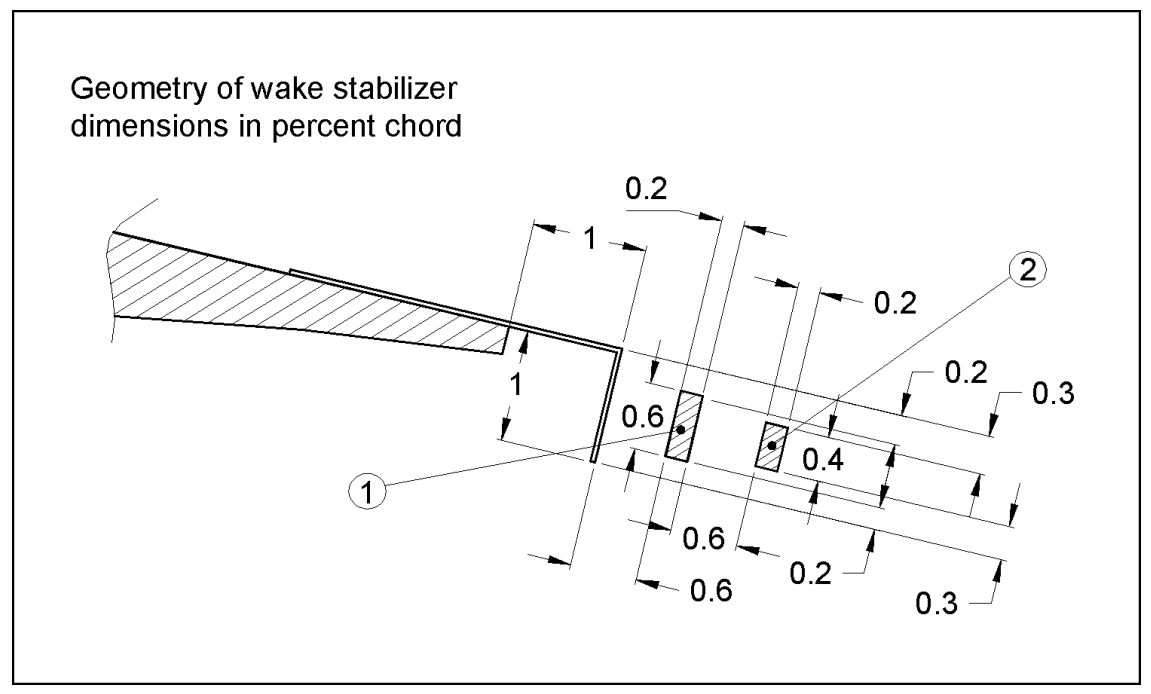

Fig. 22 Geometry of wake stabilizers $\left.{ }^{*}\right)$

Fig. 23 shows data with one stabilizer (1) only and with both stabilizers (1)+(2). With both stabilizers being, present, the device drag reduction is $22 \%$, whereas with merely one stabilizer, it is only $15 \%$. As we will see later, the insertion of bodies into the wake flow offers even much more significant improvements.

\footnotetext{
*) Accidentally, the lower-fairing at the trailing edge upstream of the Gurney flap had been omitted. However, this does not change the data perceivably, and certainly not towards a lower drag.
} 

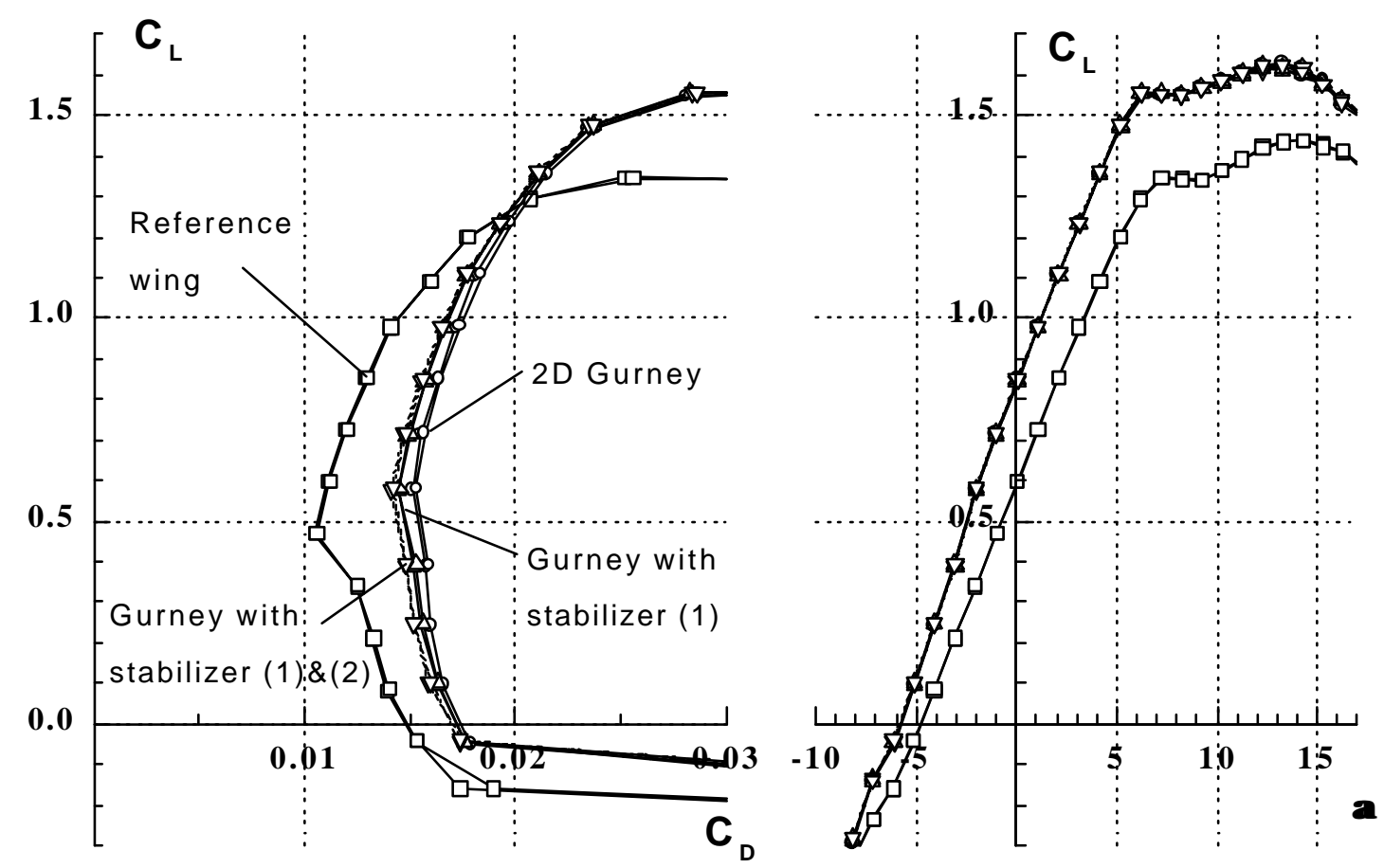

\begin{tabular}{|c|l|c|c|c|c|c|}
\cline { 3 - 6 } \multicolumn{2}{l|}{} & $\begin{array}{c}\mathrm{h} \\
{[\%]}\end{array}$ & $\begin{array}{c}\Delta \mathrm{h} \\
{[\%]}\end{array}$ & $\mathrm{C}_{\mathrm{L} \max }$ & $\mathrm{C}_{\mathrm{D} \min }$ & $\varepsilon_{\max }=\left[\frac{C_{L}}{C_{D}}\right]_{\max }$ \\
\hline$-\square-$ & Reference wing & 0.33 & - & 1.438 & 0.0105 & 69.6 \\
\hline$-0-$ & 2D Gurney flap & 1 & 0.67 & 1.627 & 0.0151 & 63.6 \\
\hline$-\Delta-$ & $\begin{array}{l}\text { Gurney flap } \\
\text { with stabilizer (1) }\end{array}$ & 1 & 0.67 & 1.623 & 0.0144 & 64.4 \\
\hline$-\nabla-$ & $\begin{array}{l}\text { Gurney flap } \\
\text { with stabilizer (1)\&(2) }\end{array}$ & 1 & 0.67 & 1.622 & 0.0141 & 64.7 \\
\hline
\end{tabular}

Fig. 23. Data of Gurney flap with wake stabilizer

\subsection{Gurney flaps with rib stiffeners.}

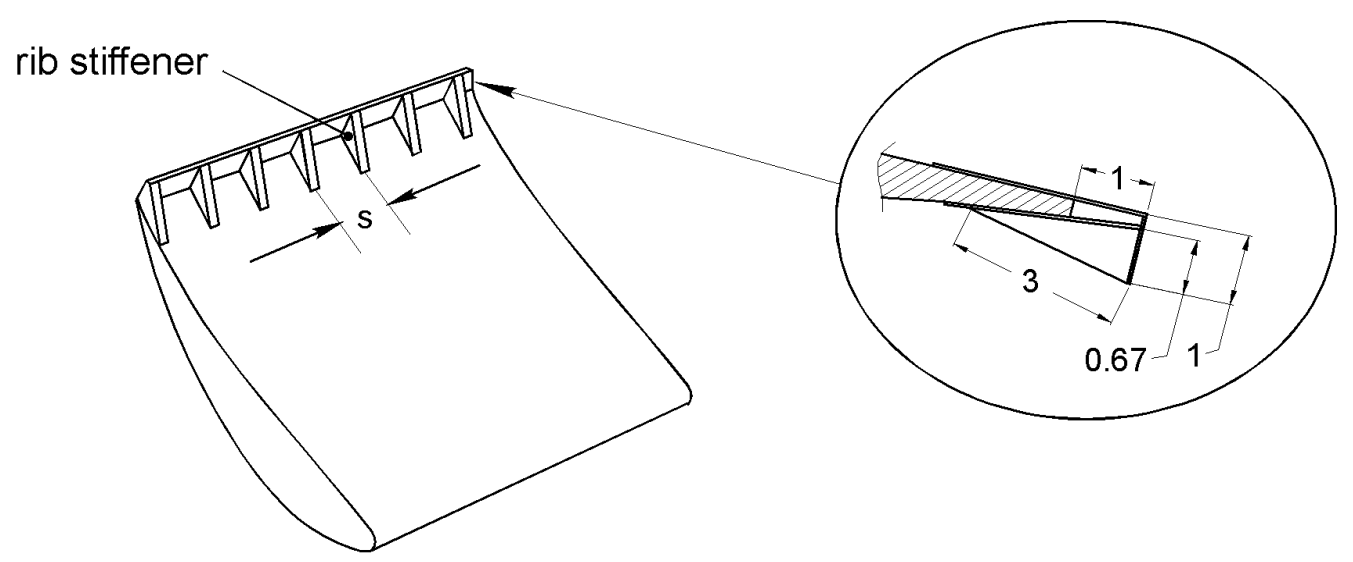

Fig. 24. Configuration of the rib stiffeners. Lateral spacings $s=2$ and $4 \%$ chord.

The basic idea of this configuration was to perturb three-dimensionally the shear flow which is shed from the lower edge of the Gurney flap. We have tested two geometries with two different lateral spacings, see Fig. 24. The desired wake stabilization and drag reduction, however, could not be observed. Probably, the three-dimensional flow perturbation upstream of the Gurney flap edge was just too small. On the other hand, a device drag increase was not observed either. Therefore, in order to increase the mechanical stiffness of the trailing edge, this device may well be used in combination with real drag-reducing modifications like, e.g., holes in the Gurney flap. 


\subsection{Trailing edge pattern of dragonfly wings.}

The authors were wondering whether or not Gurney flaps were absent in flying animals. In the paper by Okamoto, Yasuda \& Azuma [18] cross sections of dragonfly wings are shown which clearly hint at the existence of those devices in Nature. Dragonflies are among the fastest and most agile flying insects. Brackenbury in his book on "Insects in Flight" [19] refers to dragonflies as "the embodiment of speed, power and control in flight". Recently, we have learned much more about dragonfly wings from Dr. Antonia Kesel (University of Saarbruecken) [20]. Not only that Gurney flap-like shapes of wing trailing edges can be observed on dragonflies, there is a truly intriguing three-dimensional structure on the wing trailing edges. Fig. 25 shows microscope photographs of these structures taken by Dr. Kesel.

In most flying insects, only unsteady wing motion occurs, but dragonflies do also glide with motionless wings. Obviously the Reynolds numbers of dragonfly wings are low $\left(\operatorname{Re}<10^{4}\right)$. At those low Reynolds numbers, it is difficult to obtain appreciable lift coefficients. Thus, a Gurney flap-like trailing edge structure is likely to help.

In the meantime, Dr. Kesel has carried out low Reynolds number wind tunnel experiments which clearly show that indeed an increase of lift can be achieved with trailing edges emulating those observed on dragonfly wings. We assume that an absolute instability in the wake of the Gurney flap of the dragonfly occurs, even at those low Reynolds numbers. Therefore, we interpret the dark spade-shaped elements in Fig. 25 as vortex generators which destroy the two-dimensionality of the Gurney flap wake and in turn eliminate the absolute instability with its ensuing Karman vortex street. Probably, as in our higher Reynolds number experiments, that reduces the drag.

We were wondering whether or not a dragonfly-like trailing edge geometry may have a beneficial effect even at high Reynolds numbers. Therefore, we carried out measurements at $\operatorname{Re}=0.5-1 \times 10^{6}$. The trailing edge geometry can be seen in Fig. 26. It is close to what we find on dragonfly wings (Fig. 25). The shape is manufactured by electric discharge machining out of thin metal plates. We tested one "dragonfly" trailing edge with the spade-like structures sticking out perpendicularly (1) and another configuration where the spade-like structures were inclined at an angle of $45^{\circ}$ (2), see Fig. 26 . Surprisingly, the "dragonfly" trailing edge worked as well as the Gurney flaps with slits or holes. The data with a $45^{\circ}$ spade angle exhibit, as expected, a slightly decreased drag. Incidentally, with the spadelike structures only attached to the lower edge of the Gurney flap, no significant beneficial effect is found.

The real dragonfly wing (Fig. 25) exhibits spade-like structures where the upward pointing and the downward pointing spades are not always located in the same spanwise position, i.e., they are not always opposite to each other. Therefore, we have also tested whether or not shifting the spades laterally to each other has an influence. It turns out that in the drag polars a difference between both cases, shifted and not shifted, cannot be discerned. The hot wire measurements (not shown here) indicate that the wake stabilization of the dragonfly trailing edge is very good. The single frequency in the fluctuation spectrum is completely cancelled, actually slightly more efficiently for the case where the spades on both sides are not shifted relative to each other.

The mechanical stiffness of this "dragonfly" trailing edge design is very good. For comparison, a trailing edge with slits would not be advantageous for the dragonfly, because the mechanical stiffness would lack. One still wonders how evolution in several hundred million years arrived at this solution and, whether or not, as is usual in nature, there might be additional purposes for which this particular structure may have evolved. 
(a)

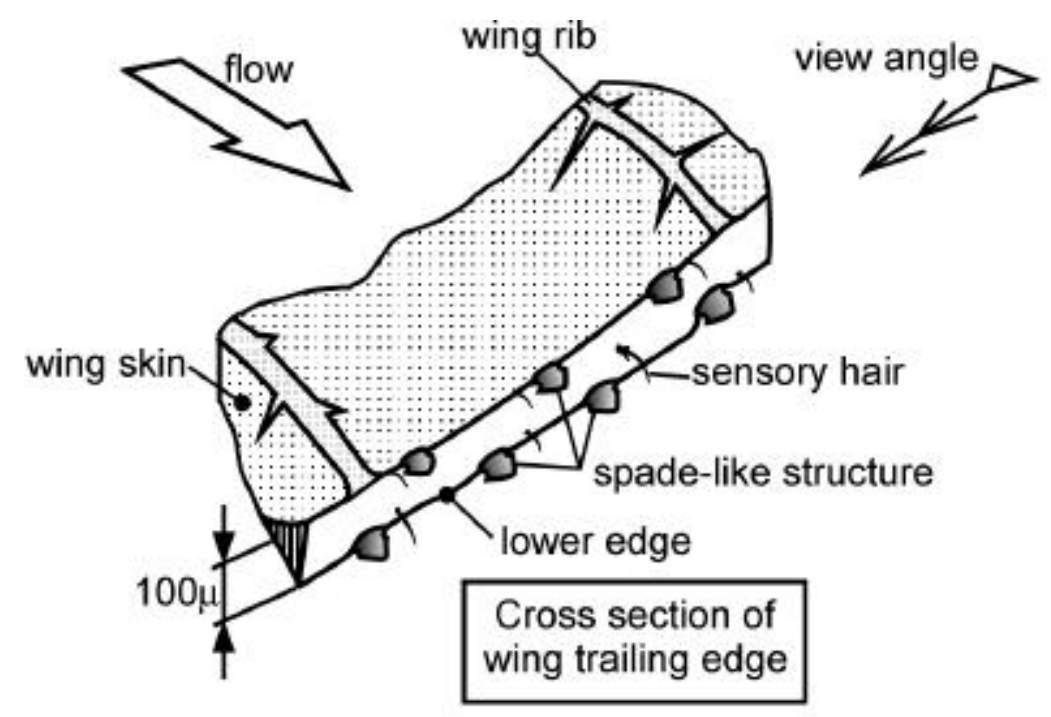

(b)

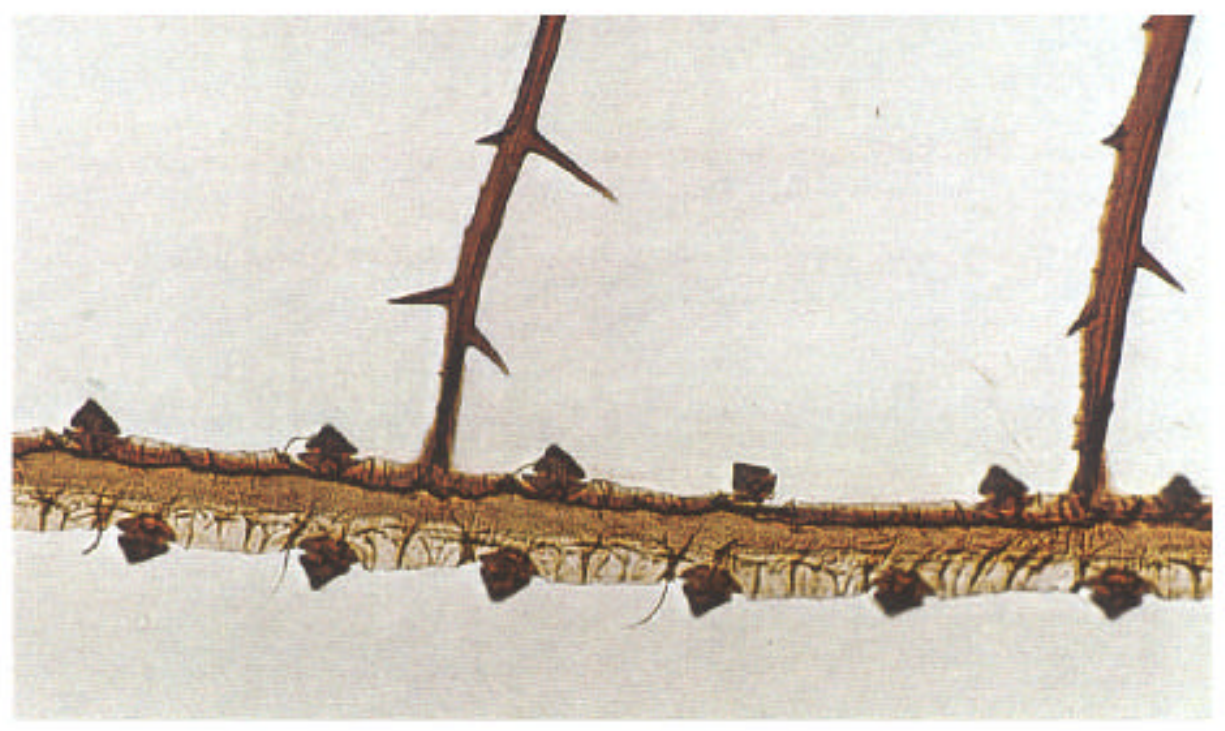

(c)

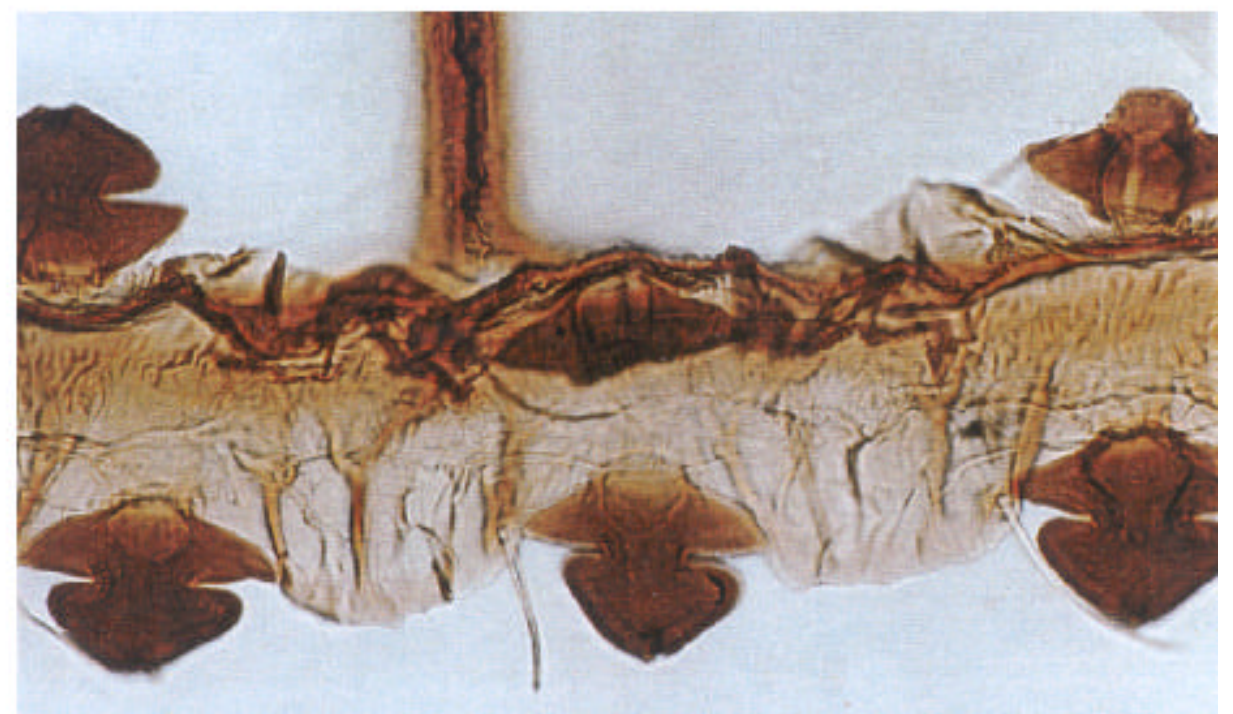

Fig. 25. Trailing edge of dragonfly wing. Photographs by A. Kesel [20]

(a) sketch indicating view angle

(b) wing trailing edge

(c) magnified details of trailing edge. 


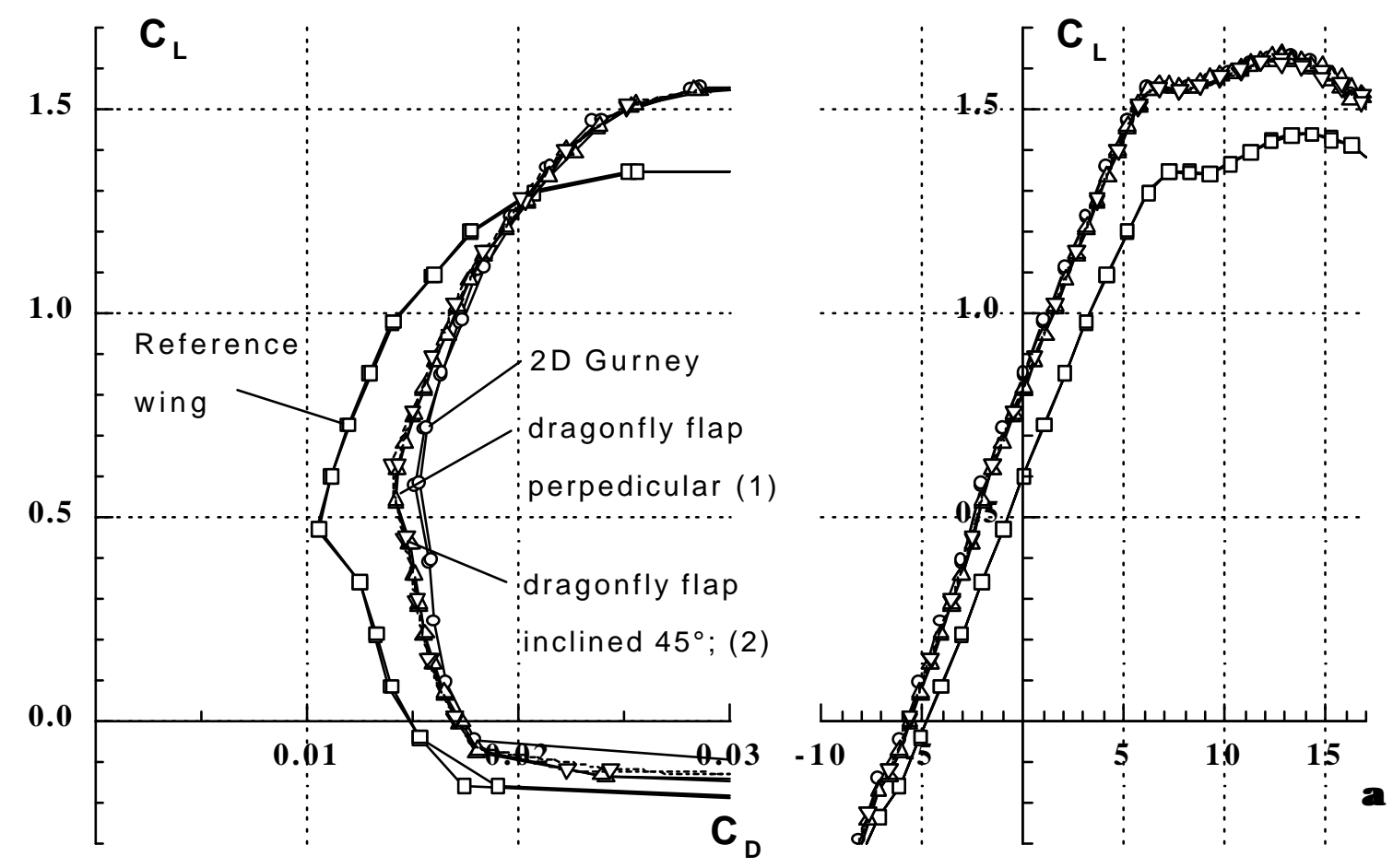

\begin{tabular}{|c|l|c|c|c|c|c|}
\cline { 3 - 7 } \multicolumn{2}{l|}{} & $\begin{array}{c}\mathrm{h} \\
{[\%]}\end{array}$ & $\begin{array}{c}\Delta \mathrm{h} \\
{[\%]}\end{array}$ & $\mathrm{C}_{\mathrm{L} \max }$ & $\mathrm{C}_{\mathrm{D} \min }$ & $\varepsilon_{\max }=\left[\frac{C_{L}}{C_{D}}\right]_{\max }$ \\
\hline$-\square-$ & Reference wing & 0.33 & - & 1.438 & 0.0105 & 69.6 \\
\hline$-0-$ & 2D Gurney flap & 1 & 0.67 & 1.627 & 0.0151 & 63.6 \\
\hline$-\Delta-$ & $\begin{array}{l}\text { dragonfly flap. } \\
\text { perpendicular (1) }\end{array}$ & 1 & 0.67 & 1.634 & 0.0142 & 62.9 \\
\hline$-\nabla-$ & $\begin{array}{l}\text { dragonfly flap. } \\
\text { inclined 45 (2) }\end{array}$ & 1 & 0.67 & 1.622 & 0.0140 & 63.7 \\
\hline
\end{tabular}

Geometry of artifical dragonfly flap, dimensions in percent chord
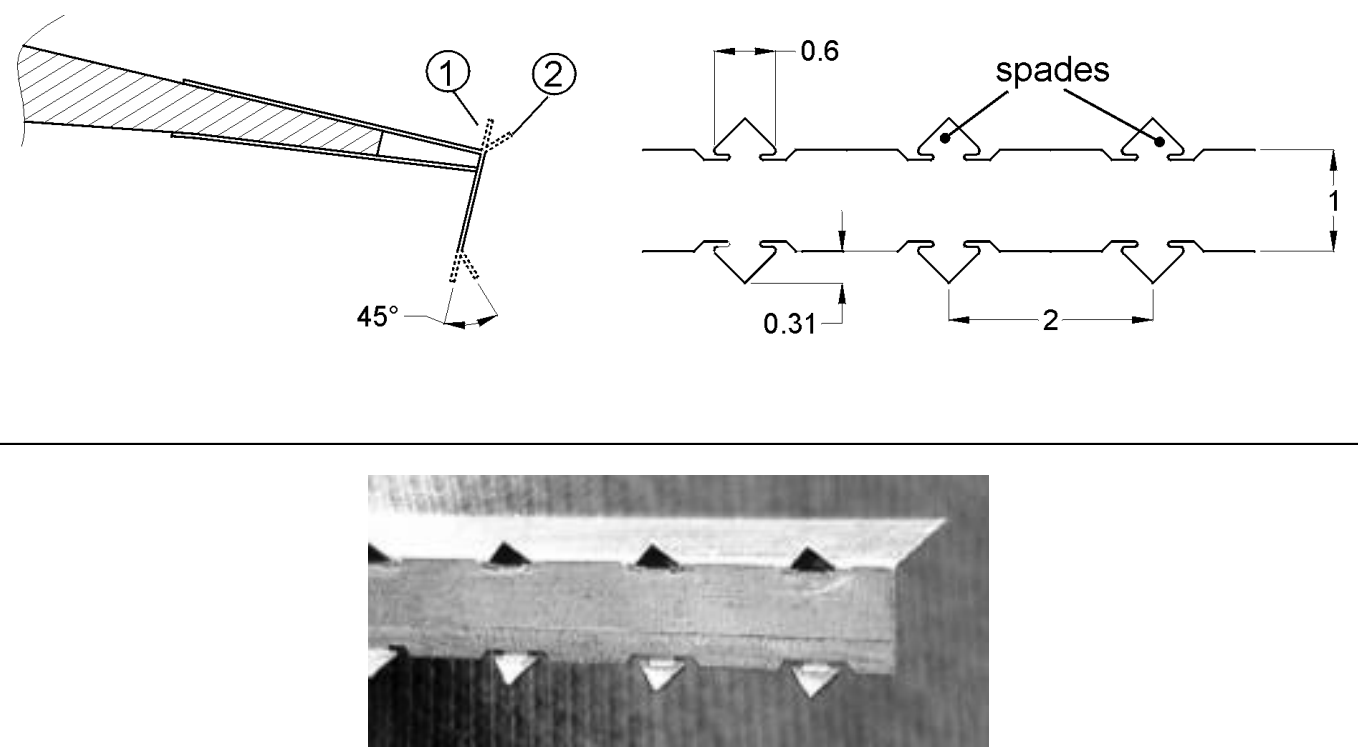

Fig. 26. Drag polars and geometrical configuration of the "dragonfly" trailing edge. 


\section{Drag reduction with a "wake body".}

Thus far, we have considered only such devices which stabilize the wake flow. Obviously, also a suitably designed splitter plate $[22,23,5]$ may be an option to achieve that. However, a much better performance can be expected from a device which eliminates the separation downstream of the Gurney flap altogether. This can be achieved with a tapered two-dimensional body downstream of the Gurney flap. We have coined the term "wake body" for this device.

In the following, we will describe the way by which we arrived at that particular shape of the "wake body" which we finally tested:

(i) In the separation regime downstream of the Gurney flap, there is a slightly decreased static pressure [5]. This lower static pressure is assumed to be generated by the entrainment of the comparatively fast flow of the shear layer leaving the lower edge of the Gurney flap. In this way, a weak suction is exerted on the flow leaving the upper surface of the airfoil. Because we consider this as a beneficial effect, we try to keep it by having a gap between "wake body" and Gurney flap.

(ii) Because a re-circulating flow is expected to occur in this gap, we inserted a vertical blade into the gap. This should ensure a sufficient suppression of the re-circulating flow there. Thus the occurrence of a local absolute instability should be avoided.

(iii) The tapering angle of the wake body should be small enough to permit attached flow on both sides. The leading edges of the body are rounded in order to prevent separation if the adjustment of the shape to the flow might not be optimal.

(iv) The exact location and the angle at which the wake body should be installed was not completely clear to us in the beginning. Therefore, we used a Drela-code calculation [14] to gain a perception of the angle at which the wake leaves from the trailing edge of the airfoil. In addition, we built a prototype wake body which could pivot freely to its equilibrium position downstream of the Gurney flap for each angle of attack of the airfoil. It turned out that the changes of the equilibrium angle of the pivoting wake body amounted to only a few degrees for the relevant operation range of the airfoil. Thus, we fixed the angle of the wake body to that condition where the best lift to drag ratio of the airfoil (with Gurney flap) was expected.

Although the above selection procedure for the shape of the wake body cannot as yet be considered optimal, the results are, nevertheless impressive, see Fig. 27. The data exhibit a device drag reduction of $48 \%$ and more, depending on the selected angle of attack.

However, once we close the gap between Gurney flap and wake body with a gap sealing, we find that the lift is reduced (as expected) and that the drag is also slightly reduced. This loss in lift proves that indeed there is a suction flow through the (open) gap as indicated in Fig. 27.

For comparison with the fixed wake body, we have also collected drag polars for a wake body which could pivot freely. The data turned out to be not better than those of a fixed wake body. By the way, the movable wake body did not vibrate or flutter unless very high angles of attack were reached where the flow on the upper side of the wing was completely separated. As a conclusion, we consider therefore a simple fixed wake body as a good choice. 

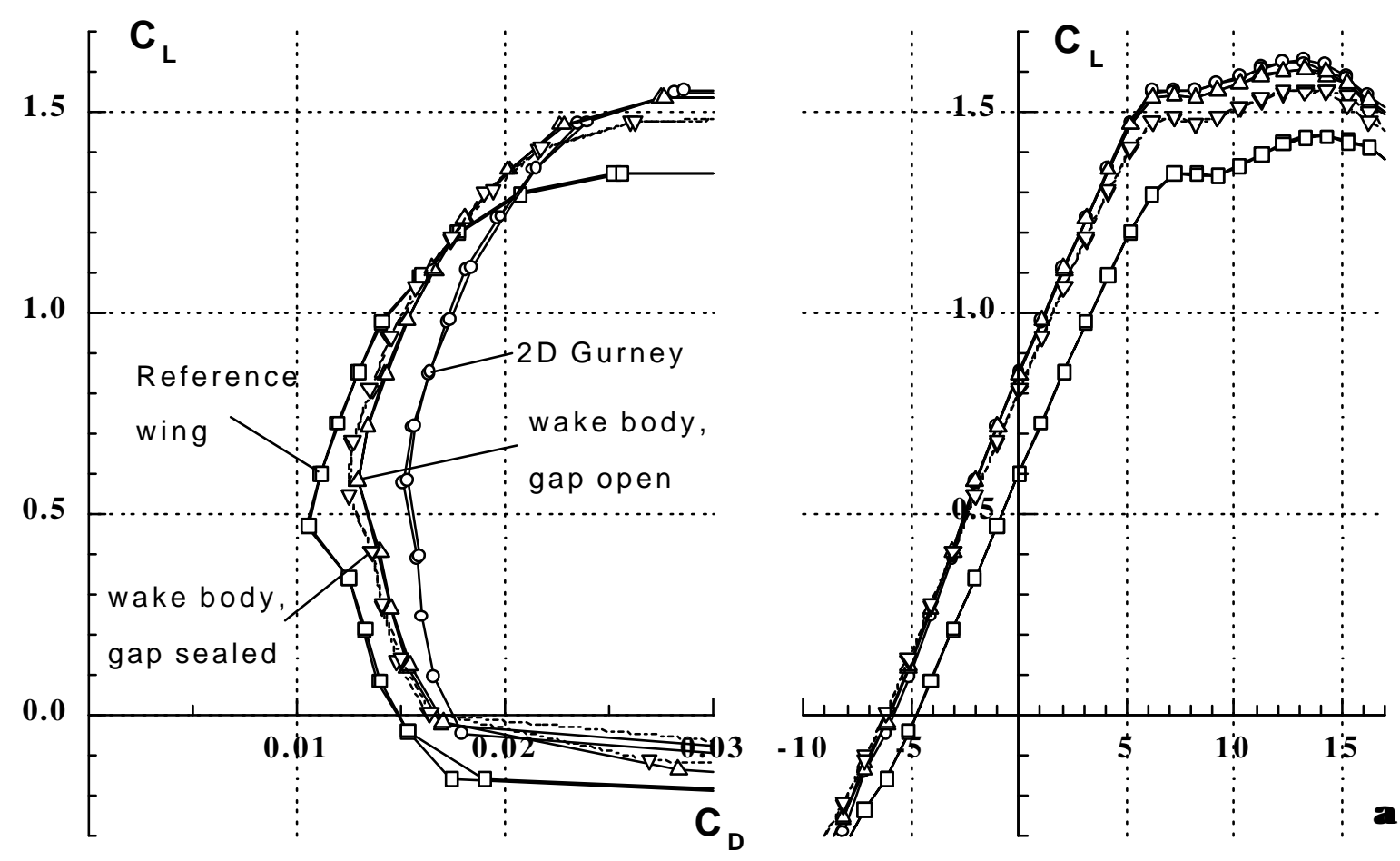

\begin{tabular}{|c|l|c|c|c|c|c|}
\cline { 3 - 7 } \multicolumn{2}{l|}{} & $\begin{array}{c}\mathrm{h} \\
{[\%]}\end{array}$ & $\begin{array}{c}\Delta \mathrm{h} \\
{[\%]}\end{array}$ & $\mathrm{C}_{\mathrm{L} \max }$ & $\mathrm{C}_{\mathrm{D} \min }$ & $\varepsilon_{\max }=\left[\frac{C_{L}}{C_{D}}\right]_{\max }$ \\
\hline$-\square-$ & Reference wing & 0.33 & - & 1.438 & 0.0105 & 69.6 \\
\hline$-0-$ & 2D Gurney flap & 1 & 0.67 & 1.627 & 0.0151 & 63.6 \\
\hline$-\Delta-$ & $\begin{array}{l}\text { with wake body } \\
\text { gap open (a) }\end{array}$ & 1 & 0.67 & 1.605 & 0.0129 & 68.6 \\
\hline$-\nabla-$ & $\begin{array}{l}\text { with wake body } \\
\text { gap sealed (b) }\end{array}$ & 1 & 0.67 & 1.551 & 0.0125 & 68.4 \\
\hline
\end{tabular}

(a) gap open

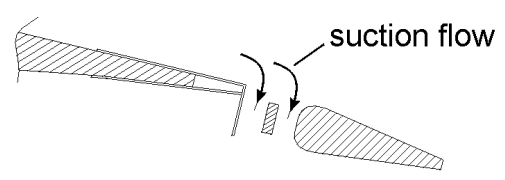

Geometry of wake body with stabilizer, dimensions in percent chord length

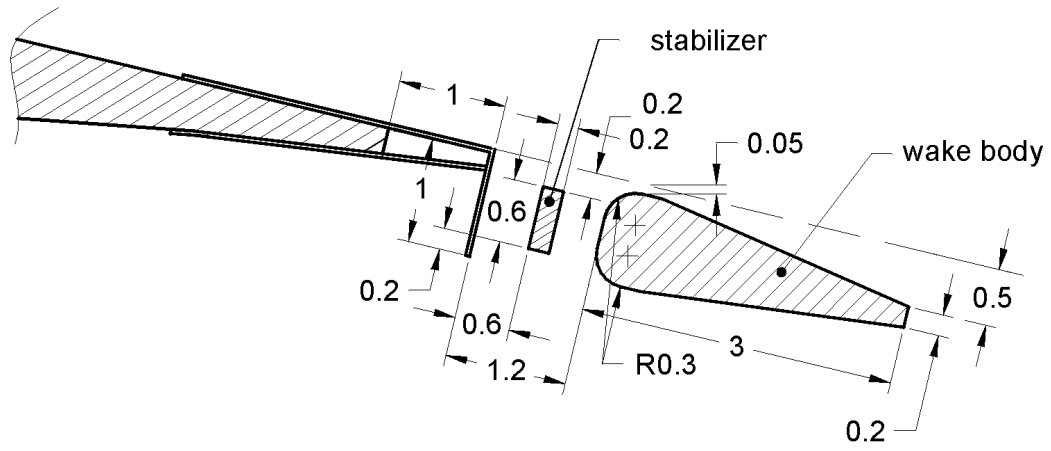

Fig. 27. Performance of the wake body. For comparison, also data are shown with the gap between Gurney flap and wake body being sealed. All coefficients are normalised without taking the additional wing area into account which is contributed by the wake body. Taking that into account would not change the lift to drag ratio, but both the lift and drag coefficients would be slightly reduced. 


\section{Acknowledgement}

the various parts of this research were supported by different contributors. The measurements on a transonic wing were carried out together with Dr. E. Stanewsky, DLR Göttingen. The suggestion to carry out these measurements came from Dr. J. Mertens, DaimlerChrylser Aerospace Airbus, Bremen. This company together with the German Federal Ministry of Research and Technology (BMBF) provided the funding. The low-speed measurements were carried out in close co-operation with the Institute of Fluid Mechanics (Hermann-Föttinger-Institut) of the Technical University of Berlin. Funding was provided by the Volkswagen Foundation and by the German National Science Foundation (Deutsche Forschungsgemeinschaft) in the Special Research Activity (Sonderforschungsbereich 557) on Control of Turbulent Shear Flows. We are particularly grateful to Dr. Antonia Kesel (University of Saarbruecken) for providing novel information and photographs of dragonfly wings.

\section{References}

[1] Liebeck, R. H. 1978, Design of subsonic airfoils for high lift. Journal of Aircraft, Vol. 15, No. 9, Sept., pp. $547-561$.

[2] Henne, P. A. 1990, Innovation with computational aerodynamics: The divergent trailing edge airfoil. In: Applied Computational Aerodynamics (Ed. P. A. Henne), Progress in Astronautics and Aeronautics, Vol. 125, AIAA, Washington

[3] Pailhas, G., Sauvage, P., Touvet, Y. \& Coustols, E. 1998, Flow field in the vicinity of a thick cambered trailing edge. Paper presented at the $9^{\text {h }}$ International Symposium on Applications of Laser Techniques to Fluid Mechanics, 13 - 16 July, Lisbon, Portugal.

[4] Coustols, E., Pailhas, g. \& Sauvage, P. 1999, Scrutinizing flow field pattern around thick cambered trailing edges: Experiments and computations. Paper presented at the $4^{\text {th }}$ International Symposium on Engineering Turbulence Modelling and Measurements, 24 - 26 May, Corsica, France.

[5] Sauvage, P. 1995, Ph.D. Thesis, École nationale Supérieure de l'aeronautique et de l'espace, Toulouse.

[6] Ross, J. C., Storms, B. L. \& Carrannanto, P. G. 1995, Lift-enhancing tabs on multielement airfoils. Journal of Aircraft, Vol. 32, No. 3, May/June, pp. 649 - 655.

[7] Kentfield, J. A. C.. 1994, Theoretically and experimentally obtained performances of Gurney flap equipped wind turbines. Wind Engineering, Vol. 18, No. 2, pp. $63-74$.

[8] Stanewsky, E. 1981, Wechselwirkung zwischen Aussenstroemung und Grenzschicht an transsonischen Profilen. Dissertation, Technische Universität Berlin.

[9] Bechert, D. W., Stanewsky, E. \& Hage, W. 1999, Windkanalmessungen an einem TranssonikFluegel mit stroemungsbeeinflussenden Massnahmen. Teil 1: Polaren, Teil 2: Druckverteilungen. DLR-IB 223-99 C05; IB 92517-99/B3-1 \& 2.

[10] Bechert, D. W., Bruse, M., Hage, W., Meyer, R. 1997, Biological surfaces and their Technological Application . Laboratory and Flight Experiments on Drag Reduction and Separation Control, AIAA-Paper 97-1960.

[11] Rae, W. H. Jr. \& Pope, A. 1984, Low-speed wind tunnel testing, Wiley, New York.

[12] Boermans, L. M. M. 1995, Wind tunnel data of the HQ17/14.38 airfoil, Delft University of Technology, Faculty of Aerospace Engineering.

[13] Althaus, D. 1996, Niedrig-Geschwindigkeits-Profile, Vieweg-Verlag, Braunschweig.

[14] Drela, M. 1996, "Xfoil" 6.8. An analysis and design system for airfoils. MIT, Dept. Of Aeronautics and Astronatics. E-mail: drela@ henry.mit.edu.

[15] Bechert, D. W. 1985, Excitation of instability waves, Zeitschrift für Flugwissenschaften und Weltraumforschung, Vol. 9, Nov/Dez, Heft 6, pp. 356 - 361.

[16] Koch, W. 1985, Local instability characteristics and frequency determination of self-excited wake flows. Journal of Sound and Vibration, Vol. 99, pp. $53-83$.

[17] Huerre, P. \& Monkewitz, P. 1985, Absolute and convective instabilities in free shear layers. Journal of Fluid Mechanics, Vol. 159, pp. $151-168$.

[18] Okamoto, M., Yasuda, K. \& Azuma, A. 1996, Aerodynamic characteristics of the wings and body of a dragonfly. The Journal of Experimental Biology, Vol. 199, pp. $281-294$. 
[19] Brackenbury, J. 1995, Insects in flight, Blandford, Casell, London.

[20] Kesel, A. 2000, Material, Struktur, Funktion. Die ultraleichten Tragflächen der Insekten (Material, structure, function: The ultra light wings of insects) VDI-Kunststoffband 4225: Plastics in automotive engineering, Jahrestagung der VDI-Gesellschaft Kunststofftechnik, Mannheim 5 - 6 April. In addition: personal communication Dept. of Zoology, Technical Biology \& Bionics, University of Saarland, Saarbruecken.

[21] Wakeling, J. M. \& Ellington, C. P. 1997, Dragonfly flight, Parts I-III, The Journal of Experimental Biology, Vol. 200, pp. $543-600$.

[22] Roshko, A. B. 1955, Bluff bodies, Journal of Aeronautical Sciences, p 124, see also: NACA Tech. Rpt. 1191 (1954) and NACA Tech. Notes 2913 (1953) and 3169 (1954).

[23] Hoerner, S. F. 1965, Fluid-dynamic drag. Edition: S. F. Hoerner, 148 Busteed Drive, Midland Park New Jersey, 07432, USA. 